\title{
Ranking multidimensional alternatives and uncertain prospects
}

\author{
Philippe Mongin ${ }^{\mathrm{a}, *}$, Marcus Pivato ${ }^{\mathrm{b}, \mathrm{c}}$ \\ ${ }^{a}$ Centre National de la Recherche Scientifique \& HEC Paris, France \\ ${ }^{\mathrm{b}}$ Department of Mathematics, Trent University, Canada \\ ${ }^{\mathrm{c}}$ THEMA, Université de Cergy-Pontoise, France
}

Received 2 April 2013; final version received 26 June 2014; accepted 31 December 2014

Available online 7 January 2015

\begin{abstract}
We introduce a ranking of multidimensional alternatives, including uncertain prospects as a particular case, when these objects can be given a matrix form. This ranking is separable in terms of rows and columns, and continuous and monotonic in the basic quantities. Owing to the theory of additive separability developed here, we derive very precise numerical representations over a large class of domains (i.e., typically not of the Cartesian product form). We apply these representations to (1) streams of commodity baskets through time, (2) uncertain social prospects, (3) uncertain individual prospects. Concerning (1), we propose a finite horizon variant of Koopmans's (1960) [25] axiomatization of infinite discounted utility sums. The main results concern (2). We push the classic comparison between the ex ante and ex post social welfare criteria one step further by avoiding any expected utility assumptions, and as a consequence obtain what appears to be the strongest existing form of Harsanyi's (1955) [21] Aggregation Theorem. Concerning (3), we derive a subjective probability for Anscombe and Aumann's (1963) [1] finite case by merely assuming that there are two epistemically independent sources of uncertainty.
\end{abstract}

(c) 2015 Elsevier Inc. All rights reserved.

\section{JEL classification: D70; D81}

Keywords: Multiattribute utility; Separability; Subjective probability; Harsanyi; Koopmans; Ex ante versus ex post welfare

\footnotetext{
* Corresponding author.

E-mail addresses: mongin@greg-hec.com (P. Mongin), marcuspivato@gmail.com (M. Pivato).
} 


\section{Introduction and overview}

Consider the intertemporal problem in consumer theory, i.e., to define a preference over intertemporal consumption plans ranging over several goods. A convenient way to tackle this problem is to postulate two sets of more elementary preferences, the first set comprising of preferences defined on time sequences of consumption for each given good, and the second set comprising of preferences defined on goods baskets for each given time period. Then, a preference over consumption plans can be defined by the condition that it varies ceteris paribus in the same direction as each elementary preference in either set.

Now suppose that a social observer wants to compare social prospects, which allocate either money or utility across both individuals and states of the world. This can be dealt with as before, by first supposing two sets of elementary preferences, and then requiring that the overall preference agree ceteris paribus with them. Here, one set is obtained by fixing the individual and letting the states vary, and the other, by fixing the state and letting the individuals vary. That is, the observer judges the social prospects both from all possible ex ante individual perspectives, and from all possible ex post social perspectives, with his final judgment taking each such judgment separately into account.

Changing the model again, suppose that an individual decision-maker has to compare statecontingent prospects, where each state of nature encapsulates information from two epistemically independent ${ }^{1}$ sources of uncertainty. Then the same technique as before leads one to introduce two sets of conditional preferences over the consequences, and have the preference over prospects reflect any of these conditionals ceteris paribus.

Each time, the objects are structured in terms of two attributes, and an overall preference is obtained by assuming that it is separable in terms of the values taken by either attribute. Separability has become a familiar theme in economics at large, but we aim at exploring it further, both abstractly and in terms of applications. Our main interest lies with social preference under uncertainty, as we will show - following some unpublished work by the first author (Blackorby et al. [2]) - that advanced separability techniques deliver surprisingly powerful results in this area. However, the theory needed there proves to be so general as to cover many other cases at once, and it is a secondary goal of this paper to bring out this rich potential. Thus, we review the intertemporal consumer preference problem, and introduce the mixed uncertainty problem, using the same toolkit to gain insight on them. The three cases have to do with defining preferences of some kind, but we could have investigated some non-preferential rankings at little extra cost. ${ }^{2}$

Before proceeding, we briefly sketch the main technical ideas of the paper. In general, the alternatives take the form of matrices of real numbers, with the indices of rows and columns representing two independent attributes of these objects, such as commodities and moments in time, individuals and states of nature, or two independent sources of uncertainty. ${ }^{3}$

If there are more than two attributes, one could group them within the row indices or within the column indices, so as to apply the matrix form. For example, in an application to intertemporal social preferences with uncertainty, each column may represent both a particular state of

\footnotetext{
1 This means that neither source of uncertainty provides any information about the other source.

2 An earlier version of the paper had one such example. It constructed an index of economic integration based on input-output matrices.

3 If the attributes exhibit some kind of logical or physical interdependency, then the matrix form is prima facie inadequate, but a redescription can sometimes make them independent. Multiattribute decision theory has considered this redescription strategy; see, e.g., Keeney [22].
} 
nature and a particular moment in time. However, this move is sometimes ill-judged, because it locates part of the aggregation process at the definitional level instead of the axiomatic one, and it precludes some pertinent representations from being derived. A more relevant alternative would be to replace matrices by higher-dimensional arrays. Our techniques easily generalize to this setting, but this will not be pursued here.

We assume that objects are ranked as follows. Each row index is associated with a ranking of those rows which are feasible given that index, and likewise, each column index is associated with a ranking of the feasible columns for that index. The overall ranking of feasible matrices relates to these rankings monotonically, i.e., if two matrices differ only in one row, and one matrix has this row ranked above the corresponding row of the other, then the first matrix is higher than the second in the overall ranking, and similarly with columns instead of rows. These are the separability conditions illustrated above. We need another crucial condition, to the effect that two matrices differing in only one coordinate (i.e., row-column pair) are ranked as the numbers in that coordinate. This is a monotonicity assumption, saying in effect that the numbers measure something valuable.

These three axioms - called Row Preferences, Column Preferences and CoordiNATE MonOtONiCiTy - often become familiar once the application context is fixed. We also impose CONTINUITY on the overall ranking. Under domain assumptions to be spelled out below, this axiom set delivers a representation theorem of a classic format: the overall ranking of matrices can be represented by a fully additively separable value function, i.e., a sum of value functions defined for each coordinate (Proposition 1 in Section 2). This functional form was axiomatized by Debreu [10] and Gorman [17], and it has since then pervaded microeconomic theory (see Blackorby, Primont, and Russell [4]) and multiattribute decision theory (see Fishburn [11], Keeney and Raiffa [24], Wakker [38]). However, it is not obtained here in the same way as in these works, and we dispense with the strong assumption, made both by Debreu and Gorman, that the set of alternatives is a full Cartesian product. To generalize this assumption, we rely on topological concepts that have been introduced first in mathematical decision theory. ${ }^{4}$ Our aim here is to take account of those feasibility constraints which our applications will typically involve.

Another important connection is with the early microeconomic literature on consistent aggregation; see Green [18], and van Daal and Merkies [34,35] for surveys; the pioneering result is due to Nataf [29]. Once translated into numerical representations, our four axioms are seen to entail consistent aggregation, which is known from this literature to entail additive separability. However, our theory goes farther by dispensing with two unpalatable assumptions of these earlier works: first, the Cartesian product structure, and second, the differentiability of numerical representations. The latter assumption precludes one from stating a proper axiomatic basis since it has no counterpart at the preference level.

As it is reexpresses the analysis of the three opening examples, Proposition 1 shows that, for all its naturalness, this analysis is constraining and perhaps undesirable. Depending on how the additively separable form is assessed, it constitutes either a characterization theorem or an impossibility theorem. The same ambivalence underlies the main results of the paper, to be described now.

\footnotetext{
4 See the technical references in Section 2. Our paper belongs to the "topological" branch of separability theory, rather than its "algebraic" branch.
} 
These results require the overall ranking to be invariant between rows (INVARIANT Row PREFERENCES), or between columns (InVARIANT COLUMN PREFERENCES), or both at the same time. Adding relevant domain assumptions to these new preference axioms, Theorem 1 strengthens the additively separable representation of Proposition 1 into a weighted sum of value functions, where the value functions may differ only across rows, or only among columns. The case where both invariance properties hold is covered by a separate result, Corollary 1.

In terms of applications, Theorem 1 is first put to use on intertemporal preference. By adding relevant conditions in the style of Koopmans [25], Proposition 2 axiomatizes the classic discounted utility model. While Koopmans's original axioms only apply to infinite consumption streams, our variant is for finite ones.

Second and more importantly, we apply Theorem 1 to uncertain social preference. As is wellknown, when social alternatives are uncertain, social welfare criteria can have two forms, either ex ante or ex post, and the question arises whether they can be made compatible. This has been debated in welfare economics by Hammond [19], in moral philosophy by Broome [6], and in axiomatic decision theory by Mongin [26]. The widespread answer is that the two criteria become compatible only if the individuals' and the social observer's ex ante preferences obey stringent restrictions. However, this conclusion depends on the prior assumption that the individuals and the social observer satisfy the axioms of expected utility theory, and little is known on the compatibility problem when this major assumption is relaxed. Because the decision-theoretic properties encapsulated in our axioms are so weak - merely statewise dominance and state-independence Theorem 1 shows what happens in this case. Somewhat shockingly, the conclusion remains negative: the same stringent conditions are necessary to achieve compatibility between the ex ante and ex post normative viewpoints.

A related connection is with Harsanyi's [21] Aggregation Theorem, which states that a Paretian and von Neumann-Morgenstern (VNM) aggregate of individual VNM utility functions is a weighted sum of these utility functions. Viewed in this light, Theorem 1 generalizes Harsanyi by showing that much weaker decision-theoretic assumptions suffice for his conclusion. In the end, an expected utility representation turns out to be indispensable, as our theorem deduces it at the same time as the weighted sum rule, so this is another ambivalent finding. On the one hand, we reinforce Harsanyi's intriguing argument for utilitarianism, by starting from better assumptions, and on the other, we establish once and for all that his argument cannot live outside of the narrow framework of linear decision theory. From a comparison with the literature, this appears to be the strongest form of the Aggregation Theorem to date.

Lastly, we apply Corollary 1 to derive a subjective probability from preferences under mixed uncertainty. Given our finiteness assumptions, Anscombe and Aumann [1] provide the relevant benchmark. They have been criticized for resorting to VNM preferences over "objective" lotteries in their derivation, and our proposed alternative avoids this classic circularity objection entirely. This result is the only the beginning of an analysis of subjective probability that will be pursued elsewhere.

\section{Basic framework and a preliminary result}

We fix two finite sets of indices, $I$ and $J$, with $|I|,|J| \geq 2$, in order to represent the relevant attributes of the objects to be ranked. These are identified with bundles of quantities $x_{j}^{i}$ for all $(i, j) \in I \times J$, and accordingly, we define an alternative $\mathbf{X}$ to be an element of the Cartesian product $\mathbb{R}^{I \times J}$. We usually write $\mathbf{X}$ in matrix form, i.e., $\mathbf{X}=\left[x_{j}^{i}\right]_{j \in J}^{i \in I}$, but sometimes also as a vector of rows or as a vector of columns. Writing $I=\{1, \ldots, n\}$ and $J=\{1, \ldots, m\}$, we put 
$\mathbf{X}=\left(\mathbf{x}^{1}, \mathbf{x}^{2}, \ldots, \mathbf{x}^{n}\right)$, where for each $i \in I, \mathbf{x}^{i}:=\left[x_{j}^{i}\right]_{j \in J} \in \mathbb{R}^{J}$ is the $i$ th row vector of $\mathbf{X}$. We also write $\mathbf{X}=\left(\mathbf{x}_{1}, \ldots, \mathbf{x}_{m}\right)$, where for each $j \in J, \mathbf{x}_{j}:=\left[x_{j}^{i}\right]^{i \in I} \in \mathbb{R}^{I}$ is the $j$ th column vector.

We assume that feasibility constraints restrict the set of alternatives. For a number of reasons, it may be impossible to realize all and every distribution of quantities through time periods, individuals, and states of the world. E.g., future consumption of a durable depends on how much it is used now, social benefits to some individuals depend on much the others earn, crops depends on the state of weather. To cover many cases by a single hypothesis, we take the set offeasible alternatives to be an open, connected subset $\mathcal{X} \subseteq \mathbb{R}^{I \times J}$. This is in line with Segal [32], Chateauneuf and Wakker [9]; still in accord with them, more topological restrictions on $\mathcal{X}$ will be introduced later.

We introduce a preference relation $\succeq$ on $\mathcal{X}$ rather than the whole of $\mathbb{R}^{I \times J}$, thus departing from the common procedure in microeconomics of divorcing preferences from constraints entirely. The common procedure is appropriate when constraints only restrict the availability of objects to the individuals, and not also their existence, since otherwise preferences would sometimes compare impossible objects between themselves or with possible objects, and this seems to be nonsensical. Our applications involve borderline cases between availability and existence. Compare elementary consumer theory, in which alternatives are static commodity baskets and can be left unrestricted unproblematically, with its intertemporal extension, which replaces them by consumptions plans and should take account of inter-period complementarities. If the conceptual difference is often overlooked, this is, we submit, on grounds of mathematical expediency: universal domains take the form of Cartesian products, which are much easier to handle than restricted sets of alternatives such as the present $\mathcal{X}$.

All of our axioms relate to $\succeq$ as a single primitive term. Throughout, we take it to be an order (meaning a weak order, i.e., a transitive and complete binary relation) and to satisfy the following four axioms. Define the projected sets $\mathcal{X}^{i}:=\left\{\mathbf{x}^{i} ; \mathbf{X} \in \mathcal{X}\right\}$, for all $i \in I$, and $\mathcal{X}_{j}:=\left\{\mathbf{x}_{j} ; \mathbf{X} \in \mathcal{X}\right\}$, for all $j \in J$.

Continuity: The preference order $\succeq$ is a continuous (i.e., its upper and lower contour sets are closed subsets of $\mathcal{X}$ ).

Row PREFERENCES: For all $i \in I$, there is an order $\succeq^{i}$ on $\mathcal{X}^{i}$ such that, for all $\mathbf{X}, \mathbf{Y} \in \mathcal{X}$, if $\mathbf{x}^{h}=\mathbf{y}^{h}$ for all $h \in I \backslash\{i\}$, then $\mathbf{X} \succeq \mathbf{Y}$ if and only if $\mathbf{x}^{i} \succeq^{i} \mathbf{y}^{i}$.

Column Preferences: For all $j \in J$, there is an order $\succeq_{j}$ on $\mathcal{X}_{j}$ such that, for all $\mathbf{X}, \mathbf{Y} \in \mathcal{X}$, if $\mathbf{x}_{k}=\mathbf{y}_{k}$ for all $k \in J \backslash\{j\}$, then $\mathbf{X} \succeq \mathbf{Y}$ if and only if $\mathbf{x}_{j} \succeq_{j} \mathbf{y}_{j}$.

Coordinate Monotonicity: For all $i \in I$ and $j \in J$, and all $\mathbf{X}, \mathbf{Y} \in \mathcal{X}$ with $x_{k}^{h}=y_{k}^{h}$ for all $(h, k) \in I \times J \backslash\{(i, j)\}, \mathbf{X} \succeq \mathbf{Y}$ if and only if $x_{j}^{i} \geq y_{j}^{i}$.

The last axiom is a consequence of either of the following conditions:

Row Monotonicity: For all $i \in I$ and $j \in J$, and all $\mathbf{x}, \mathbf{y} \in \mathcal{X}^{i}$, with $x_{k}=y_{k}$ for all $k \in$ $J \backslash\{j\}, \mathbf{x} \succeq^{i} \mathbf{y}$ if and only if $x_{j} \geq y_{j}$.

Column Monotonicity: For all $j \in J$ and $i \in I$, and all $\mathbf{x}, \mathbf{y} \in \mathcal{X}_{j}$, with $x^{h}=y^{h}$ for all $h \in I \backslash\{i\}, \mathbf{x} \succeq_{j} \mathbf{y}$ if and only if $x^{i} \geq y^{i}$.

The following lemma states this precisely, and also identifies a convenient case in which an equivalence holds. 
Lemma 1. Let $\mathcal{X} \subseteq \mathbb{R}^{I \times J}$ be an open set, and let $\succeq$ be an order on $\mathcal{X}$ that has COLUMN PREFERENCES and ROW PREFERENCES. If $\succeq$ satisfies either ROW MONOTONICITY or COLUMN MONOTONICITY, then $\succeq$ satisfies COORDINATE MONOTONICITY. Conversely, if $\mathcal{X}$ is convex, then COORDINATE MONOTONICITY is equivalent to each of ROW MONOTONICITY and COLUMN MONOTONICITY.

(The proofs of Lemma 1 and all other results are in Appendix A.)

Coordinate Monotonicity, Row Monotonicity and Column Monotonicity say that the $x_{j}^{i}$ are amounts of some good: the more of it at $(i, j)$, every other quantity being the same, the more satisfied the preference.

Row PREFERENCES and COLUMn PREFERENCES call for comparison with the more familiar condition of weak separability. ${ }^{5}$ Here is how it would occur in the present framework.

Weak Row Separability: For all $i \in I$, for all $\mathbf{X}, \mathbf{Y}, \tilde{\mathbf{X}}, \tilde{\mathbf{Y}} \in \mathcal{X}$ such that $\mathbf{x}^{i}=\tilde{\mathbf{x}}^{i}, \mathbf{y}^{i}=\tilde{\mathbf{y}}^{i}$, and for all $h \in I \backslash\{i\}$, if $\mathbf{x}^{h}=\mathbf{y}^{h}$ and $\tilde{\mathbf{x}}^{h}=\tilde{\mathbf{y}}^{h}$, then $\mathbf{X} \succeq \mathbf{Y}$ if and only if $\tilde{\mathbf{X}} \succeq \tilde{\mathbf{Y}}$.

Weak Column Separability: For all $j \in J$, for all $\mathbf{X}, \mathbf{Y}, \mathbf{X}^{\prime}, \mathbf{Y}^{\prime} \in \mathcal{X}$ such that $\mathbf{x}_{j}=\mathbf{x}_{j}^{\prime}, \mathbf{y}_{j}=\mathbf{y}_{j}^{\prime}$, and for all $k \in J \backslash\{j\}$, if $\mathbf{x}_{k}=\mathbf{y}_{k}$ and $\mathbf{x}_{k}^{\prime}=\mathbf{y}_{k}^{\prime}$, then $\mathbf{X} \succeq \mathbf{Y}$ if and only if $\mathbf{X}^{\prime} \succeq \mathbf{Y}^{\prime}$.

To clarify the connection, note first that Row PREFERENCES (RP) entails Weak Row Separability (WRS) on any domain $\mathcal{X}$. For the converse, suppose that WRS holds, and for each $i \in I$, define the following binary relation $\succeq^{i}$ :

$x^{i} \succeq^{i} y^{i} \quad$ iff there are $\mathbf{X}, \mathbf{Y} \in \mathcal{X}$

such that $\quad \mathbf{x}^{h}=\mathbf{y}^{h} \quad$ for all $h \in I \backslash\{i\}$, and $\mathbf{X} \succeq \mathbf{Y}$.

If $\mathcal{X}=\mathbb{R}^{I \times J}$, then $\succeq^{i}$ satisfies RP. However, on a domain $\mathcal{X} \varsubsetneqq \mathbb{R}^{I \times J}, \succeq^{i}$ can fail the completeness property of an ordering, and in this case, RP is strictly stronger than WRS. We need RP precisely to balance the unusual generality of our domain assumption. Barring this fine logical point, it has the same significance as WRS. This holds similarly for COLUMN PREFERENCES and Weak Column Separability.

We will now briefly review the axioms in the light of our three applications. In the intertemporal preference problem, take the sets $I$ and $J$ to represent time periods and goods, respectively. Thus, with the $x_{j}^{i}$ measuring physical quantities, ROW PREFERENCES gives rise to preferences over goods baskets at each given time, and COLUMN PREFERENCES, to preferences over consumption streams for each given good.

In the uncertain social preference problem, the sets $I$ and $J$ will represent individuals and states of nature, respectively. We can take the $x_{j}^{i}$ to be physical quantities, as in the previous case, or to be utility values, which conceptually amounts to endorsing a welfarist form of normative economics. ${ }^{6}$ We consider the latter interpretation because it connects better with the theoretical issues highlighted in the introduction. Thus, what the social preference $\succeq$ ranks are ex ante social allocations viewed in utility terms, and ROW PREFERENCES corresponds to the following Pareto conditions: (a) if all individuals are indifferent between two social prospects, then the

\footnotetext{
5 In multiattribute decision theory, see Keeney and Raiffa [24, Chap. 3]. In individual decision theory, see Fishburn [11] and Wakker [38].

6 In normative economics, welfarism is the claim that individual utility values capture all the information on alternatives that may be relevant to the social evaluation.
} 
social preference also is; (b) if one individual strictly prefers one prospect to the other, and all others are indifferent, then the social preference ranks the former prospect above the latter. Statement (a) is exactly the ex ante Pareto Indifference condition. Statement (b) is a weaker form of the ex ante Strict Pareto condition. To get the full force of it, i.e., to cover the case of more than one non-indifferent individual, one must apply (b) iteratively, and this requires a rich enough domain. Ours is intentionally small, and ROW PREFERENCES can only yield a local form of the ex ante Strict Pareto axiom. ${ }^{7}$ Thus, the ex ante Pareto principle holds in a somewhat weakened way.

COLUMN PREFERENCES means that the ex ante social preference $\succeq$ is increasing with respect to each of its ex ante preferences conditional on states. This is statewise dominance, a virtually universal property for decisions under uncertainty; it is satisfied not only by expected utility, but also by most current non-expected utility models (including rank-dependent utility). Since the $x_{j}^{i}$ are utility numbers, Row MonOTONICITY means statewise dominance for each individual order $\succeq^{i}$ vis-à-vis its own conditionals. COLUMN MONOTONICITY means that in every realized state, the ex post social preference order satisfies both Pareto Indifference and a local version of Strict Pareto. This is the ex post Pareto principle, again in a weaker form due to the domain.

In the mixed uncertainty problem, the sets $I$ and $J$ correspond to the two independent sources of uncertainty, while $x_{j}^{i}$ represents the utility payoff if the uncertainty resolves to the state of nature $(i, j)$. ROW PREFERENCES and COLUMN PREFERENCES say that preferences exist conditionally on each $i$ and conditionally on each $j$, and that the preference $\succeq$ over prospects is increasing with respect to each of these conditionals - an eventwise dominance property, since $i$ and $j$ now count as properties of states, hence as events (they are identified with $\{i\} \times J$ and $I \times\{j\}$ respectively).

We now move to more technical assumptions, which are essential to the proofs. For all $\mathbf{Y} \in \mathcal{X}$, and all $i \in I$ and $j \in J$, the $(i, j)$-section of $\mathcal{X}$ through $\mathbf{Y}$ is the set $\left\{\mathbf{X} \in \mathcal{X} ; x_{j}^{i}=y_{j}^{i}\right\}$, an $(I \cdot J-1)$-dimensional subset of $\mathbb{R}^{I \times J}$. We say $\mathcal{X}$ is sectionally connected if each $(i, j)$-section is connected. This condition is neither stronger nor weaker than connectedness; see the examples by Segal [32], Wakker [37], and Chateauneuf and Wakker [9], which also illustrate why this is an important restriction. The open set $\mathcal{X} \subseteq \mathbb{R}^{I \times J}$ is connected if and only if it is path-connected, which means that, given any two feasible alternatives $\mathbf{X}$ and $\mathbf{Y}$, it is possible continuously to transform $\mathbf{X}$ into $\mathbf{Y}$ by moving along a continuous path of feasible alternatives. Sectional connectedness resembles path-connectedness, except that it requires one to transform $\mathbf{X}$ into $\mathbf{Y}$ while holding constant the value of one coordinate. The set $\mathcal{X}$ is both path-connected and sectionally connected if it is convex and, a fortiori, if it is a box-i.e., $\mathcal{X}=\prod_{i \in I} \prod_{j \in J} \mathcal{B}_{j}^{i}$, where $\mathcal{B}_{j}^{i}$ is a real interval for all $i \in I$ and $j \in J$. (However, an open box-shaped domain would not usefully restrict the universal domain $\mathcal{X}=\mathbb{R}^{I \times J}$.)

Finally, we say that $\mathcal{X}$ is $\succeq$-indifference connected if, for all $\mathbf{Y} \in \mathcal{X}$, the indifference set $\{\mathbf{X} \in \mathcal{X} ; \mathbf{Y} \approx \mathbf{X}\}$ is a connected subset of $\mathcal{X}$. The above writers have well-explained why this restriction matters. Here are two cases in which it holds.

(a) If $\mathcal{X}$ is an open box in $\mathbb{R}^{I \times J}$, then $\mathcal{X}$ is $\succeq$-indifference connected. (See Appendix A for proof.)

$\overline{7}$ That is: for any $\mathbf{X} \in \mathcal{X}$, there is an open neighborhood $\mathcal{Y}_{\mathbf{X}} \subseteq \mathcal{X}$ with $\mathbf{X} \in \mathcal{Y}_{\mathbf{X}}$ such that, for any $\mathbf{Y} \in \mathcal{Y}_{\mathbf{X}}$ with $\mathbf{x}^{i} \succeq^{i} \mathbf{y}^{i}$ for all $i \in I$, and $\mathbf{x}^{i} \succ^{i} \mathbf{y}^{i}$ for some $i \in I$, it is the case that $\mathbf{X} \succ \mathbf{Y}$. However, if $\mathcal{X}$ is convex, one can take $\mathcal{Y}_{\mathbf{X}}=\mathcal{X}$ for all $\mathbf{X} \in \mathcal{X}$. 
(b) Suppose $\mathcal{X}$ is a convex and comprehensive subset of $\mathbb{R}_{+}^{I \times J}$. If $\succeq$ is quasiconcave, then $\mathcal{X}$ is $\succeq$-indifference connected. ${ }^{8}$

For all $i \in I$ and $j \in J$, let $\mathcal{X}_{j}^{i}:=\left\{x_{j}^{i} ; \mathbf{X} \in \mathcal{X}\right\} \subseteq \mathbb{R}$. Now to our first result.

Proposition 1. Let $\mathcal{X} \subseteq \mathbb{R}^{I \times J}$ be open. Let $\succeq$ be an order on $\mathcal{X}$ that has Row PREFERENCES and Column Preferences, and satisfies Continuity and CoOrdinate Monotonicity. Then:

(a) For all $\mathbf{X} \in \mathcal{X}$, there is an open neighborhood $\mathcal{Y} \subseteq \mathcal{X}$ with $\mathbf{X} \in \mathcal{Y}$, and for all $i \in I$ and $j \in J$, there are continuous increasing functions $u_{j}^{i}: \mathcal{X}_{j}^{i} \longrightarrow \mathbb{R}$ such that $\succeq$ is represented on $\mathcal{Y}$ by the additive function $U: \mathcal{Y} \longrightarrow \mathbb{R}$ defined by

$$
U(\mathbf{Y}):=\sum_{i \in I} \sum_{j \in J} u_{j}^{i}\left(y_{j}^{i}\right), \quad \text { for all } \mathbf{Y} \in \mathcal{Y} .
$$

Furthermore, in this representation, the $u_{j}^{i}$ are unique up to positive affine transformations with a common multiplier. ${ }^{9}$

(b) Suppose $\mathcal{X}$ is also connected, sectionally connected, and $\succeq$-indifference connected. Then we can take $\mathcal{Y}=\mathcal{X}$ in part (a).

(c) In this case, for all $i \in I$, the order $\succeq^{i}$ is represented by the function $U^{i}: \mathcal{X}^{i} \longrightarrow \mathbb{R}$ defined by

$$
U^{i}(\mathbf{x}):=\sum_{j \in J} u_{j}^{i}\left(x_{j}\right), \quad \text { for all } \mathbf{x} \in \mathcal{X}^{i} .
$$

(d) Likewise, for all $j \in J$, the order $\succeq_{j}$ is represented by the function $U_{j}: \mathcal{X}_{j} \longrightarrow \mathbb{R}$ defined by

$$
U_{j}(\mathbf{x}):=\sum_{i \in I} u_{j}^{i}\left(x^{i}\right), \quad \text { for all } \mathbf{x} \in \mathcal{X}_{j}
$$

Part (a) relies on an indirect use of Debreu's [10] theorem on additively separable representations. Instead of explicitly assuming that the preference order is totally separable, as in this classic result, we first establish total separability via the theory of overlapping separability developed in Gorman [17]. Then, Debreu's theorem provides a local additively separable representation in a box around any alternative. Part (b) consists in gluing these local representations together, via the special connectedness conditions.

We leave it for the reader to check that consistent aggregation, in Green's [18] or van Daal and Merkies's [34] sense, holds of the numerical functions representing the orders defined here. Had

\footnotetext{
$\overline{8}$ The set $\mathcal{X} \subseteq \mathbb{R}^{I \times J}$ is comprehensive if for all $\mathbf{X} \in \mathcal{X}$, and all $\mathbf{X}^{\prime} \in \mathbb{R}^{I \times J}$, if $\mathbf{X}^{\prime} \leq \mathbf{X}$ then $\mathbf{X}^{\prime} \in \mathcal{X}$. The order $\succeq$ is quasi-concave if all of its upper contour sets are convex.

9 That is, if the functions $\widetilde{u}_{j}^{i}: \mathcal{X}_{j}^{i} \longrightarrow \mathbb{R}$ are such that $\succeq$ is represented on $\mathcal{Y}$ by the function $\widetilde{U}$ defined by
}

$$
\tilde{U}(\mathbf{Y}):=\sum_{i \in I} \sum_{j \in J} \tilde{u}_{j}^{i}\left(y_{j}^{i}\right), \quad \text { for all } \mathbf{Y} \in \mathcal{Y},
$$

then there exist $a>0$ and $b_{j}^{i} \in \mathbb{R}$ such that, for all $i \in I$ and $j \in J, \tilde{u}_{j}^{i}\left(y_{j}^{i}\right)=a u_{j}^{i}\left(y_{j}^{i}\right)+b_{j}^{i}$ for all $\mathbf{Y} \in \mathcal{Y}$. 
we taken these representations as primitives, and retained Nataf's [29] strong Cartesian product and differentiability assumptions, we could have applied his theorem and obtained Proposition 1 (b) at once. ${ }^{10}$

In general, the functions $u_{j}^{i}$ are all different, and to obtain a relationship between them is the object of the following section and its more advanced results. Our applications to uncertain social preference and mixed uncertainty require these later results, but Proposition 1 already offers a perspective on intertemporal preference, as we now discuss. In this case, $u_{j}^{i}$ is a utility function for consumption of good $j$ at time $i, U^{i}$ is a utility function over consumption bundles at time $i$, $U_{j}$ is a utility function over streams of good $j$, and $U$ is a utility function for consumption plans. There is a classical stock of arguments for rejecting additive separability with respect to goods, and being suspicious of it when it applies to time periods.

Jevons and Walras introduced the "equation of exchange" - today's textbook equality between marginal utility ratios and marginal rates of substitution - in terms of separable, and even additively separable, utility functions for consumption goods, and they also stated their demand theory in this way. Edgeworth pointed out that this was unnecessary for the purpose, still a mild point, but later neo-classicals found more distressing objections. Implying as it does that the marginal rate of substitution of $a$ for $b$ only depends on the quantities of $a$ and $b$, separability (more generally than additive separability) makes the law of demand automatic under diminishing marginal utilities, thus wiping out the possibility of a prevailing income effect. Moreover, separability makes it impossible to classify consumer goods into complements and substitutes. These critical messages were taken aboard long ago by demand theory, and it comes to no surprise that postwar theorist Gorman expressed doubts about the very assumptions that he was exploring mathematically. ${ }^{11}$

Additively separable representations have on the whole been more successful when they concern time preferences. Ramsey may have been the first to employ such a functional form in his saving model, and it has persisted in the neoclassical literature on intertemporal choices of consumption, investment or money balances. This can be explained by analytical convenience, but no doubt also by the fact that the objections from demand theory lose their force here. However, there are worrying specific objections, in particular that for some goods, the quantity of today's consumption influences the utility of tomorrow's consumption through habit formation. ${ }^{12}$

Given this controversial pedigree, Proposition 1 sounds like a mixed blessing. Some might use it to axiomatize old style neo-classical economics, but many others will rather argue by contrapositive, rejecting the strong functional forms in the conclusion, and hence, the choice of the axioms in this case. The ambivalence is also typical of the results in the next section.

\section{The main theorem and its applications}

Although too strong in one sense, the conclusion of Proposition 1 is too weak in another, because the additively separable representation does not impose any relation between the utility functions defined coordinatewise. This section makes it more informative by introducing both

\footnotetext{
10 Though Nataf's [29] theorem is correct, its proof is rather obscure. The curious reader may consult the clarifications and improvements adduced by van Daal and Merkies [35].

11 More obviously in Gorman [16] than in the other papers.

12 This by now classic objection is discussed in detail by Browning [7]. Other problems raised by temporal separability are discussed in the theoretical management literature (e.g., Keeney and Raiffa [24]), as well as in health economics (e.g., Gold et al. [15]).
} 
stronger axiomatic conditions and more structural assumptions. In the axiomatic group, we will strengthen either Row PREFERENCES, or COLUMN PREFERENCES, or both, by requiring that there be a single preference order on rows, or a single preference order on columns, or both. Formally, define

$$
\mathcal{X}_{J}:=\bigcup_{j \in J} \mathcal{X}_{j} \quad \text { and } \quad \mathcal{X}^{I}:=\bigcup_{i \in I} \mathcal{X}^{i}
$$

We will require at least one of the following two conditions.

INVARIANT Row PREFERENCES: There is an order $\succeq^{I}$ on $\mathcal{X}^{I}$ such that, for all $i \in I$ and any $\mathbf{X}, \mathbf{Y} \in \mathcal{X}$ with $\mathbf{x}^{h}=\mathbf{y}^{h}$ for all $h \in I \backslash\{i\}, \mathbf{X} \succeq \mathbf{Y}$ if and only if $\mathbf{x}^{i} \succeq^{I} \mathbf{y}^{i}$.

INVARIANT COLUMN PREFERENCES: There is an order $\succeq_{J}$ on $\mathcal{X}_{J}$ such that, for all $j \in J$ and any $\mathbf{X}, \mathbf{Y} \in \mathcal{X}$ with $\mathbf{x}_{k}=\mathbf{y}_{k}$ for all $k \in J \backslash\{j\}, \mathbf{X} \succeq \mathbf{Y}$ if and only if $\mathbf{x}_{j} \succeq_{J} \mathbf{y}_{j}$.

Since our framework treats rows and columns symmetrically and their meaning can be fixed at will, we conventionally select INVARIANT COLUMN PREFERENCES when only one invariance condition applies.

In the group of structural conditions, we will require that there be a single set of feasible rows, or a single set feasible columns, or both. Formally, the domain $\mathcal{X}$ should satisfy at least one of the following structural conditions:

Identical Row Spaces: There exists $\mathcal{X}^{I} \subseteq \mathbb{R}^{J}$ such that $\mathcal{X}^{i}=\mathcal{X}^{I}$ for all $i \in I$.

Identical Column Spaces: There exists $\mathcal{X}_{J} \subseteq \mathbb{R}^{I}$ such that $\mathcal{X}_{j}=\mathcal{X}_{J}$ for all $j \in J$.

Under the first condition, there is a common projection $\mathcal{X}_{j}^{*}$ of all the $\mathcal{X}^{i}$, for each $j \in J$, with the property that $\mathcal{X}^{I} \subseteq \prod_{j \in J} \mathcal{X}_{j}^{*}$. Under the second condition, there is a common projection $\mathcal{X}_{*}^{i}$ of all the $\mathcal{X}_{j}$, for each $i \in I$, with the property that $\mathcal{X}_{J} \subseteq \prod_{i \in I} \mathcal{X}_{*}^{i}$. Here are two formal cases where the conditions hold.

Examples (a) If $\mathcal{X}$ is an open box in $\mathbb{R}^{I \times J}$, then $\mathcal{X}$ satisfies both Identical Row Spaces and Identical Column Spaces.

(b) Suppose that, for all $\mathbf{y} \in \mathcal{X}_{J}$, there exists $\mathbf{X} \in \mathcal{X}$ such that $\mathbf{x}_{j}=\mathbf{y}$ for all $j \in J$. Then $\mathcal{X}$ satisfies Identical Column Spaces.

InVARIANT Row PrefEREnCES and InVARIANT COLUmn PREFERENCES are so formulated that no logical implication holds between them and Identical Row Spaces and Identical Column Spaces. However, the two sets of restrictions are often acceptable or rejectable together. In the intertemporal preference problem, INVARIANT ROW PREFERENCES and Identical Row Spaces are very stringent, while Invariant COLUmn Preferences and Identical Column Spaces may or may not hold depending on the case. The former says that one time ranks commodity baskets like another when they are available at both times; this excludes habit formation. The latter asserts that exactly the same baskets are available at each time; this excludes exogenous changes in the feasible set over time, such as those brought about by technological innovation or climate change, but emphatically, it does not exclude technical interdependencies between periods. ${ }^{13}$ Consumer theory often makes these assumptions.

$\overline{13}$ This would rather be excluded by assuming $\mathcal{X}$ to be the Cartesian product $\prod_{j \in J} \mathcal{X}_{j}$. 
In the uncertain social preference problem, with $x_{j}^{i}$ representing utility, INVARIANT ROW PREFERENCES becomes the implausible claim that all individuals have the same preferences. But Identical Row Spaces is not so easy to discard. It says that the set of utility vectors is common to all individuals, which makes sense if some interpersonal utility comparisons have already taken place. Meanwhile, INVARIANT COLUMN PREFERENCES says that ex post social preferences are state-independent, while Identical Column Spaces says that the same social outcomes are feasible in each state. These two state-independence assumptions are made by Savage [31] and Anscombe and Aumann [1] when they derive a subjective probability from preferences under uncertainty, and they have prevailed in the theoretical discussion of ex ante versus ex post social welfare criteria that concerns us. Note that this axiom still allows the individuals to have state-dependent preferences; this is explained below.

Now to our main result. Given a finite set $K$ and a vector $\mathbf{p} \in \mathbb{R}^{K}$, we say that $\mathbf{p}$ is a strictly positive weight vector on $K$ if $p_{k}>0$ for all $k \in K$, and $\sum_{k \in K} p_{k}=1$. We reserve the expression of probability vector for those cases in which elements of $K$ represent states of nature. The set of strictly positive weight vectors on $K$ is denoted by $\Delta_{K}$.

Theorem 1. Suppose that $\mathcal{X} \subseteq \mathbb{R}^{I \times J}$ is open, connected, sectionally connected, $\succeq$-indifference connected, and satisfies Identical Column Spaces. Then $\succeq$ satisfies CONTINUITY, COORDINATE Monotonicity, Row Preferences and Invariant Column Preferences if and only if:

(a) For all $i \in I$, there is an increasing, continuous function $u^{i}: \mathcal{X}_{*}^{i} \longrightarrow \mathbb{R}$, such that the order $\succeq_{J}$ is represented by the function $W_{J}: \mathcal{X}_{J} \longrightarrow \mathbb{R}$ defined by

$$
W_{J}(\mathbf{x}):=\sum_{i \in I} u^{i}\left(x^{i}\right), \quad \text { for all } \mathbf{x} \in \mathcal{X}_{J} .
$$

(b) There is a strictly positive weight vector $\mathbf{p} \in \Delta_{J}$, such that for all $i \in I$, the order $\succeq^{i}$ is represented by the function $U_{\mathbf{p}}^{i}: \mathcal{X}^{i} \longrightarrow \mathbb{R}$ yielding the $\mathbf{p}$-weighted value of $u^{i}$. That is:

$$
U_{\mathbf{p}}^{i}(\mathbf{x}):=\sum_{j \in J} p_{j} u^{i}\left(x_{j}\right), \quad \text { for all } \mathbf{x} \in \mathcal{X}^{i} .
$$

(c) The order $\succeq$ is represented by the function $W: \mathcal{X} \longrightarrow \mathbb{R}$ which computes the $\mathbf{p}$-weighted value of the function $W_{J}$ from part (a). That is:

$$
W(\mathbf{X}):=\sum_{j \in J} p_{j} W_{J}\left(\mathbf{x}_{j}\right)=\sum_{j \in J} \sum_{i \in I} p_{j} u^{i}\left(x_{j}^{i}\right)=\sum_{i \in I} U_{\mathbf{p}}^{i}\left(\mathbf{x}^{i}\right), \quad \text { for all } \mathbf{X} \in \mathcal{X} .
$$

(d) In this representation, the weight vector $\mathbf{p}$ is unique, and the functions $u^{i}$ are unique up to positive affine transformations with a common multiplier.

Application to intertemporal preferences Suppose $J:=\{1,2, \ldots, t\}$ indexes a set of $t$ consecutive time periods. Then, Theorem 1 says that time $j$ does not influence the shape of the utility functions $u^{i}$ defined for each commodity $i$, its role being channeled through the weights $p_{j}$. To turn these weights into discount factors, more axioms are needed. Essentially, we adapt those of Koopmans [25], which were defined for infinite consumption streams, whereas we have a finite horizon $t$ here. ${ }^{14}$

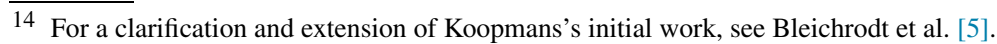


For any $\mathbf{X} \in \mathcal{X}$, let $\mathbf{X}_{L} \in \mathbb{R}^{|I| \times(t-1)}$ be the submatrix comprised of the leftmost $(t-1)$ rows of $\mathbf{X}$, and let $\mathbf{X}_{R} \in \mathbb{R}^{|I| \times(t-1)}$ be the submatrix comprised of the rightmost $(t-1)$ rows of $\mathbf{X}$. Let $\mathcal{X}_{L}:=\left\{\mathbf{X}_{L} ; \mathbf{X} \in \mathcal{X}\right\}$ and $\mathcal{X}_{R}:=\left\{\mathbf{X}_{R} ; \mathbf{X} \in \mathcal{X}\right\}$; these are both open subsets of $\mathbb{R}^{|I| \times(t-1)}$.

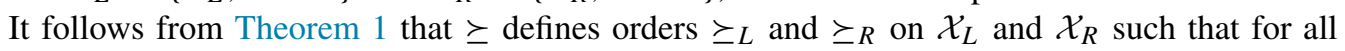
$\mathbf{X}, \mathbf{Y} \in \mathcal{X}:$

- If $\mathbf{x}_{t}=\mathbf{y}_{t}$, then $\mathbf{X} \succeq \mathbf{Y}$ iff $\mathbf{X}_{L} \succeq_{L} \mathbf{Y}_{L}$.

- If $\mathbf{x}_{1}=\mathbf{y}_{1}$, then $\mathbf{X} \succeq \mathbf{Y}$ iff $\mathbf{X}_{R} \succeq_{R} \mathbf{Y}_{R}$.

We require that these auxiliary orders coincide. This is Koopmans's stationarity condition, but adapted to a finite set of time periods.

STATIONARITY. $\succeq_{L}$ is identical with $\succeq_{R}$ on $\mathcal{X}_{L} \cap \mathcal{X}_{R}$.

We also impose a version of Koopmans's impatience condition. It says that a commodity bundle becomes preferable if it consumed at an earlier date than the current one. For all $j \in$ $\{1,2, \ldots, t-1\}$ and all $\mathbf{X} \in \mathcal{X}$, let $\sigma_{j}(\mathbf{X})$ be the matrix resulting from interchanging columns $j$ and $j+1$ in $\mathbf{X}$.

Impatience for $j$. For all $\mathbf{X} \in \mathcal{X}, \mathbf{X} \succeq \sigma_{j}(\mathbf{X}) \Longleftrightarrow \mathbf{x}_{j} \succeq_{J} \mathbf{x}_{j+1}$.

For our purposes, this condition will have enough bite if there exists $\mathbf{X} \in \mathcal{X}$ such that $\sigma_{j}(\mathbf{X}) \in \mathcal{X}$ and the $j$ and $j+1$ columns of $\mathbf{X}$ are non-indifferent for $\succeq^{J}$; in this case, we say that time $j$ is activated. Given our domain assumption and earlier axioms, this is a light requirement. ${ }^{15}$ With this added material, we can now turn the weighted sum $W(\mathbf{X})$ of Theorem 1 into a discounted sum.

Proposition 2. Suppose the hypotheses of Theorem 1 hold.

(a) Suppose that for all $j \in\{1, \ldots, t-1\}$, IMPATIENCE FOR $j$ holds and time $j$ is activated. Then, $p_{j}>p_{j+1}$ for all $j \in\{1, \ldots, t-1\}$.

(b) Suppose that for some $j \in\{1, \ldots, t-1\}$, IMPATIENCE FOR $j$ holds and $j$ is activated, and suppose also that STATIONARITY holds with $\mathcal{X}_{L} \cap \mathcal{X}_{R} \neq \emptyset$. Then, there exists some $\delta \in(0,1)$ such that $p_{j}=\delta^{j-1} \cdot p_{1}$ for all $j \in J$.

Application to uncertain social preferences The functions $U_{\mathbf{p}}^{i}$ and $W$ of Theorem $1(\mathrm{~b}, \mathrm{c})$ represent the individuals' and the social observer's ex ante utility functions. If $\mathbf{p}$ is regarded as a probability vector, then these functions are shown to be of the expected utility type. This seems to be a strong conclusion, given the modicum of decision theory we assumed at the start. We required only two things: first, that both the individuals and social observer satisfy statewise dominance, and second, that the social observer has Paretian and state-independent preferences. Theorem 1(b) does not impose state-independent preferences on the individuals, because the $x_{j}^{i}$

\footnotetext{
$\overline{15}$ For example, it is satisfied if there is $\mathbf{X} \in \mathcal{X}$ with $\mathbf{x}_{j}=\mathbf{x}_{j+1}$. (For some $\varepsilon>0, \mathcal{X}$ contains $\mathbf{X}^{(\epsilon)}$, which is $\mathbf{X}$ except that in its $j$-th column $\mathbf{x}_{j}^{(\epsilon)}, \epsilon$ is added to each component of $\mathbf{x}_{j}$. From Column Monotonicity, $\mathbf{x}_{j}^{(\epsilon)} \succ_{J} \mathbf{x}_{j+1}$.)
} 
are taken to be preexisting utility numbers which may very well come from some state-dependent utility functions, exogenous to our modeling.

Theorem 1(a, c) gives another description of the social observer's preferences, this time in terms of social welfare functions. The ex post welfare function $W_{J}$ and the ex ante welfare function $W$ are sums of the corresponding individual utility functions, i.e., have the mathematical form of a weighted utilitarian rule. This strongly cardinal conclusion seems surprising in view of the purely ordinal form of the axioms, but the separability conditions have contributed their familiar role in the inference step. ${ }^{16}$ Whether the $W_{J}$ and $W$ representations bear more than a formal analogy with classical utilitarianism is a complex question that we do not discuss here.

Finally, Theorem 1(d) confers uniqueness to the functional representations, under the usual proviso that the mathematical pattern in which they appear must be respected. ${ }^{17}$ Without such uniqueness, the representations would have no significance; in particular, it would not be sensible to view $\mathbf{p}$ as being anybody's probability.

With these interpretations at hand, Theorem 1 has a bearing on the classic problem in welfare economics of comparing ex ante with ex post criteria. By definition, the ex ante social welfare criterion applies the ex ante Pareto principle to the individuals' ex ante preferences, while assuming that these individual preferences conform to some specific decision theory (typically subjective expected utility theory). Meanwhile, the ex post social welfare criterion puts the decision-theoretic restrictions on the social preference order, while applying the ex post Pareto principle to the individuals' ex post preferences. As Section 2 explained, the four components just listed correspond to Row PREFERENCES, Row Monotonicity, COLUMn MonotonicITY and COLUMN PREFERENCES, respectively, so the assumptions of the theorem characterize a social welfare criterion that would be both ex ante and ex post. ${ }^{18}$ The conclusion shows that this hypothesized compatibility can be achieved only if

(1) the individuals and the observer are all expected utility maximizers, and

(2) they compute their expected utilities by using the same subjective probabilities.

Hammond's [19] welfare economics paper is a good source for both the compatibility problem and the answer that conclusion (2) is necessary for its solution. ${ }^{19}$ When investigating consistent ways of aggregating Savage preferences, Mongin [26] implicitly raised the compatibility problem. His axiomatic treatment enlarges the set of possibilities somewhat. The ex ante and ex post social criteria can be compatible when either weaker Pareto conditions than the full Pareto principle apply, or the individuals' utility functions are affinely dependent. These two possibilities are excluded by ROW PREFERENCES and the open domain assumption, respectively, so it comes as no surprise that only (2) survives in Theorem 1 . The main news is conclusion (1). The above papers (and others as well) assumed that both the individuals and the social observer satisfied the axioms of subjective expected utility, whereas we now prove this as a component part of our representation. To appreciate the step forward, take probabilistically sophisticated individuals,

\footnotetext{
16 The step from ordinality to cardinality through separability is well-documented in Fishburn [11] and Wakker [38].

17 Non-affine monotonic transforms of the $u^{i}$ would represent the $\succeq^{i}$ equally well, but destroy the expected utility form of the representations in Theorem $1(\mathrm{~b}, \mathrm{c})$.

18 Observe that the ex ante and ex post Pareto principles are logically independent in our framework. This is not the case in the frameworks of the next paragraph, which embody subjective expected utility assumptions. There, the ex post principle automatically follows from applying the ex ante principle to constant prospects.

19 Hammond acknowledges Starr's [33] paper on allocation under uncertainty as being an earlier source.
} 
i.e., individuals who have well-defined subjective probabilities despite not satisfying subjective expected utility, but some generalization of it. They would satisfy our weak decision-theoretic conditions; thus, if the observer satisfied both the ex ante and ex post criteria, they would inexorably turn into subjective expected utility maximizers!

It is unclear whether conclusion (2) represents an impossibility theorem or only a severe, though implementable restriction. Among the interpreters, Broome [6] seems to take the latter view, whereas Mongin and d'Aspremont [28] favour the former. The answer depends on one's underlying philosophy of probability, and on the further issue of when probabilities are computed: is it at the completely ex ante stage, or rather at some interim stage? On one interpretation, probabilities are subjective in the sense promoted by Savage, and moreover, they are pure priors, i.e., embody no outside information at all; this would make their identity across individuals rather unlikely. On another interpretation, they are still subjective in the same sense, but are imperfect priors, thus in effect posteriors, because they embody some outside information; this would make their identity across individuals less unlikely. (Some will argue that a pure prior is a fiction and that this is the only appropriate alternative of the two.) Finally, probabilities could be objective in one of the senses that philosophers of probability have argued for. ${ }^{20}$ This last interpretation would make (2) unproblematic, but it does not fit in with the present frame of analysis, which, like Savage's, take probabilities to reflect subjective preferences.

Several proposals have been made to escape from (2) when it is viewed as an impossibility. The pure ex ante solution and pure ex post solution each keep only one of the two social welfare criteria. Indeed, the ex post solution is the common way out in welfare economics. ${ }^{21}$ However, quite a few respondents have suggested that compatibility would result from weakening the decision-theoretic basis, and Theorem 1 has the clearest bearing when it comes to this group. It definitely wrecks the hopes set in replacing the sure-thing principle by one of its recent generalizations, but leaves open a less obvious possibility, which is to let the social observer have state-dependent preferences. That conciliation is forthcoming along this line has been confirmed (see Mongin [27] and Chambers and Hayashi [8]). We do not explore the solutions any further and defer to another paper explaining how our separability theory could contribute to them. ${ }^{22}$

Theorem 1 also relates to Harsanyi's [21] Aggregation Theorem. According to this classic result, if the individuals have VNM preferences on a lottery set, and if the social observer satisfies the Pareto principle and himself entertains VNM preferences on the lottery set, then his preferences can be represented by a positively weighted sum of the VNM representations of the individual preferences. Harsanyi boldly claimed utilitarian relevance for this piece of reasoning. Our framework does not contain lotteries, so in order to bridge the gap with Harsanyi, we should replace his theorem by one of the variants that were devised for state-contingent prospects instead of lotteries. ${ }^{23}$ When this is done, Theorem 1 appears to be a stronger form of the classic result: expected utility theory now belongs to the conclusions, and the utilitarian-looking social welfare functions follow from much weaker assumptions than before.

\footnotetext{
20 An interesting recent option is objective Bayesianism (see Williamson [39]).

21 See the eloquent defence in Hammond [20].

22 Mongin and d'Aspremont [28] review the solutions proposed at the time. Among the more recent writers, Gilboa et al. [14] and Keeney and Nau [23] have taken up the challenge.

23 These variants make states of the world explicit and put identical subjective probabilities on the individuals and the observer. One of them, by Mongin [26], is stated for Savage probabilities, and the other, by Blackorby et al. [3], for probabilities on any finite set of states.
} 
Some previous works have tampered with expected utility assumptions in Harsanyi's theorem, and they call for a comparison. The earliest, Blackorby et al. [2], takes a Cartesian product set of state-contingent prospects, expressed separability and monotonicity conditions similar to Row Preferences, Column Preferences, and CoORdinate Monotonicity, although stated in utility terms instead of preferences, and eventually derived an additively separable representation for social preference. At a closer look, this representation boils down to expected utility, so that this early result is subsumed by Theorem 1 as a particular case. Not so for the theorem by Gajdos et al. [13], which requires a framework in the style of Anscombe and Aumann [1]. The individual and social preferences there obey VNM independence and Savage's sure-thing principle on only a restricted set of alternatives, and they can be state-dependent. Under an appropriate Pareto condition, the conclusion (2) of a unique subjective probability emerges in more general form, and the social utility representation is expressed as a weighted sum of the individual ones. Both because of its lottery framework and residual expected utility assumptions, this result is closer to Harsanyi's than ours. Similarly with Fleurbaey's [12] theorem, which makes full-fledged expected utility assumptions on the individuals, its contribution being to weaken those made on the social observer. This theorem, unlike the two earlier ones, does not take a full domain of alternatives, but a convex set of utility values, a particular case of our domain assumptions.

Application to intertemporal social preferences With $I$ representing the individuals and $J$ the time periods, Theorem 1 becomes a statement about intertemporal social welfare. Row PREFERENCES corresponds to the Pareto principle as applied to the individuals' intertemporal preferences, Row Monotonicity to individual time-dominance, COLUMN MonOTONICITY to the Pareto principle as applied in a given time period, and COLUMN PREFERENCES to social time-dominance. The conclusion about $\mathbf{p}$ can be strengthened by adding the conditions of Proposition 2; it will then exactly mean that the individuals share the same discount factor. As before, this may be interpreted as either a sheer impossibility or only a severe restriction, and we lean towards the former view.

It remains to examine the case in which the four conditions introduced by this section jointly apply. If $\mathcal{X}$ has both Identical Column Spaces and Identical Row Spaces, there is a single open subset $\mathcal{X}_{*}^{*}$ such that $\mathcal{X}_{j}^{i}=\mathcal{X}_{*}^{*}$ for all $(i, j) \in I \times J$.

Corollary 1. Suppose $\mathcal{X} \subseteq \mathbb{R}^{I \times J}$ is open, connected, sectionally connected, $\succeq$-indifference connected, and has Identical Row Spaces and Identical Column Spaces. Then $\succeq$ satisfies CONTINUITY, COORDINATE MONOTONICITY, INVARIANT Row PREFERENCES and INVARIANT COLUMN PREFERENCES if and only if there is an increasing, continuous function $u: \mathcal{X}_{*}^{*} \longrightarrow \mathbb{R}$ and strictly positive weight vectors $\mathbf{q}=\left(q^{i}\right)_{i \in I} \in \Delta_{I}$ and $\mathbf{p}=\left(p_{j}\right)_{j \in J} \in \Delta_{J}$ such that:

(a) The order $\succeq_{J}$ is represented by the function $W_{J}: \mathcal{X}_{J} \longrightarrow \mathbb{R}$ defined by

$$
W_{J}(\mathbf{x}):=\sum_{i \in I} q^{i} u\left(x^{i}\right), \quad \text { for all } \mathbf{x} \in \mathcal{X}_{J} .
$$

(b) The order $\succeq^{I}$ is represented by the function $W^{I}: \mathcal{X}^{I} \longrightarrow \mathbb{R}$ defined by

$$
W^{I}(\mathbf{x}):=\sum_{j \in J} p_{j} u\left(x_{j}\right), \quad \text { for all } \mathbf{x} \in \mathcal{X}^{I} .
$$


(c) The order $\succeq$ is represented by the function $W: \mathcal{X} \longrightarrow \mathbb{R}$ defined by

$$
W(\mathbf{X}):=\sum_{j \in J} \sum_{i \in I} q^{i} p_{j} u\left(x_{j}^{i}\right), \quad \text { for all } \mathbf{X} \in \mathcal{X} .
$$

(d) In this representation, the weight vectors $\mathbf{q}$ and $\mathbf{p}$ are unique, and the function $u$ is unique up to a positive affine transformation.

Application to individual intertemporal choice under uncertainty With $I$ representing a set of time periods and $J$ a set of states of nature, an element of $\mathcal{X}$ becomes an intertemporal, state-dependent prospect. Elements of $\mathcal{X}^{I}$ represent instantaneous prospects (which, by Identical Row Spaces, could be realized at any moment in time), while elements of $\mathcal{X}_{J}$ represent ex post consumption streams (which, by Identical Column Spaces, could be realized in any state of nature). INVARIANT ROW and COLUMN PREFERENCES mean, respectively, that preferences are state-independent over ex post consumption streams, and time-independent over instantaneous prospects. Adding the conditions of Proposition 2 to Corollary 1, we conclude that the individual's preference is represented by the expected value of a discounted utility sum.

An axiomatization of subjective probability for mixed uncertainty A classic objection to Anscombe and Aumann [1] is that they miss their target - i.e., to provide non-probabilistic foundations of the use of the probability calculus - since they postulate well-understood VNM lotteries to start with. The equally classic rejoinder is that lotteries represent objective probability measures, whereas the endogenous derived probability measure is subjective, and that these are markedly different concepts. However, this is a somewhat dubious response, because nothing in the framework justifies taking lotteries, and especially all possible ones, as if they were objective probabilities in a serious sense, and even frequencies, as some have suggested. A reinterpretation of Corollary 1 suggests a way out of this controversy.

We now conceive of the Cartesian product set $I \times J$ as being the set of states of nature. That is, we suppose that each state of nature is realized after two uncertainties, here represented by $I$ and $J$, are resolved. A matrix $\mathbf{X} \in \mathbb{R}^{I \times J}$ becomes a prospect that assigns a real-valued pay-off (say a monetary prize) to each state $(i, j) \in I \times J$. The preference relation $\succeq$ on prospects can be restricted to a feasible subset $\mathcal{X} \subseteq \mathbb{R}^{I \times J}$, a generalization that standard expected utility theories do not offer. ROW PREFERENCES and COLUMN PREFERENCES now define preferences conditional the $I$ - and $J$-attributes of a state, and they make an eventwise dominance claim in either case. CoORdinATE MONOTONICITY adds a statewise dominance claim with respect to the full states.

The two specific axioms of this section, i.e., INVARIANT Row PREFERENCES and INVARIANT COLUMn PREFERENCES, play the decisive role. They express the fact that the two sources of uncertainty are epistemically independent of each other in the following sense: information about how one of them is resolved reveals nothing about how the other would be. ${ }^{24} \mathrm{~A}$ semiformal argument will explain this connection. Suppose that $\xi>\xi^{\prime}$, and that contrary to the desired property, event $i \in I$ tends to make event $j \in J$ more likely to occur than event $k \in J$, whereas event $h \in I$ has the opposite tendency of making $k$ more likely to occur than $j$. Now, consider $\mathbf{X} \in \mathcal{X}$ such that $x_{j}^{i}=\xi, x_{j}^{h}=\xi^{\prime}, x_{k}^{i}=\xi^{\prime}, x_{k}^{h}=\xi$, and $\mathbf{Y} \in \mathcal{X}$ such that $y_{j}^{i}=\xi^{\prime}$,

\footnotetext{
24 To parody Savage, suppose I am uncertain whether I will find fresh eggs on the market and whether I have a bowl in my cupboard, or I am uncertain whether there will be a swimming pool open and whether my tennis racket is good enough to play.
} 
$y_{j}^{h}=\xi, y_{k}^{i}=\xi, x_{k}^{h}=\xi^{\prime}$, with $\mathbf{X}$ and $\mathbf{Y}$ sharing their values elsewhere. By the axioms of the previous paragraph, conditional preferences are well-defined and increasing, so that $x^{i} \succ^{i} y^{i}$ and $x^{h} \succ^{h} y^{h}$, contradicting INVARIANT ROW PREFERENCES since $x^{h}=y^{i}$ and $y^{h}=x^{i}$. Similarly, if $j \in J$ makes $i \in I$ more probable than $h \in I$, while $k \in J$ has the opposite effect of making $h$ more probable than $i$, a contradiction results for INVARIANT COLUMN PREFERENCES. Thus, if the two invariance conditions hold, the assumed violations of independence will not occur. This suggests that the conditions do capture independence in the sought after sense.

We assume Identical Row Spaces and Identical Column Spaces, but this is only for technical simplicity.

Let us now consider the weight vectors $\mathbf{p}$ and $\mathbf{q}$ in the conclusions of Corollary 1. Given their role in $W_{J}$ and $W^{I}$, and their uniqueness property, they express meaningful probabilities for each uncertainty. Moreover, their multiplicative occurrence in the formula

$$
W(\mathbf{X})=\sum_{j \in J} \sum_{i \in I} q^{i} p_{j} u\left(x_{j}^{i}\right)
$$

shows that the informal concept of independence has been turned into probabilistic independence. Beside answering the problem of axiomatizing this numerical concept in decisiontheoretic terms, Corollary 1 connects with the initial controversy about Anscombe and Aumann. One may retain their basic insight, which is to derive the agent's probabilities on subjectively uncertain states from preference comparisons that involve a second uncertain phenomenon. But unlike Anscombe and Aumann, we do not assume that the agent has vNM preferences with respect to this second phenomenon, or even assume that the second phenomenon admits "objective" probabilities at all. It is enough to require independence in the informal sense explained above.

\section{Conclusion}

The paper has developed a theory for ranking multiattribute alternatives that relies on the earlier Debreu-Gorman apparatus of separability, but extends it in several ways. Not only have we made this apparatus compatible with restricted feasible sets, but we have turned its generic additively separable representations into more expressive ones, which relate more closely to the aimed at applications. Those covered here are sufficient to illustrate the logical power of the theory, but others will be developed elsewhere for their own sake. Even in the field of normative economics, where the theory originates and has its currently major application, there seems to be more room for concrete work. We may put GDP time-series, income or wealth distributions, or systems of interpersonal utility comparison into suitable matrix forms, and then check whether or not our domain restrictions and axiomatic conditions meaningfully apply. Some of these cases might raise loss of dimensionality problems, and not all them will accommodate the special invariant preference and identical spaces axioms that enhanced our treatment, so the forthcoming applications are likely to range all the way down from the generic additive separability result à la Debreu-Gorman in Section 2 to the specific ones in Section 3. The theory as such deserves to be developed beyond the case of two attributes, and it would have to be generalized to the case in which the components of the matrices - or the higher-dimensional arrays - are not real numbers. 


\section{Acknowledgments}

The authors are grateful to the editors and a referee for their active guidance. They have also benefited from comments made by Chris Chambers, Eric Danan, Thibault Gajdos, Peter Hammond, Bob Nau, Peter Wakker, and audiences at seminars and conferences, when the paper was presented at the London School of Economics, the Australian National University, the Exeter Business School, the Ecole normale supérieure, the Université de Cergy-Pontoise, the 2013 PET and Francqui Prize conferences, and the 2014 RUD and D-TEA conferences. The first author gratefully acknowledges support from the ANU when he was visiting there in 2013. The second author acknowledges support from NSERC grant \#262620-2008. This paper was written while the second author was at Trent University.

\section{Appendix A. Proofs}

Proof of Lemma 1. Clearly, Row Monotonicity or Column Monotonicity imply Coordinate Monotonicity. We show the nontrivial converse. Suppose $\mathcal{X}$ is convex, and satisfies Coordinate Monotonicity; we will show that it satisfies Column Monotonicity. Let $j \in J$ and $i \in I$, and let $\mathbf{x}, \mathbf{y} \in \mathcal{X}_{j}$. Suppose $x^{h}=y^{h}$ for all $h \in J \backslash\{i\}$; we must show that $\mathbf{x} \succeq_{j} \mathbf{y}$ if and only if $x^{i} \geq y^{i}$.

Case 1. First suppose $\mathcal{X}$ is a box. Then we can find $\widetilde{\mathbf{X}}, \widetilde{\mathbf{Y}} \in \mathcal{X}$ such that $\widetilde{\mathbf{x}}_{j}=\mathbf{x}$ and $\widetilde{\mathbf{y}}_{j}=\mathbf{y}$, while $\widetilde{\mathbf{y}}_{k}=\widetilde{\mathbf{x}}_{k}$ for all $k \in J \backslash\{j\}$. Thus, we have:

$$
\left(\mathbf{x} \succeq_{j} \mathbf{y}\right) \quad \Longleftrightarrow \quad(\widetilde{\mathbf{X}} \succeq \tilde{\mathbf{Y}}) \quad \Longleftrightarrow \quad\left(\tilde{x}_{j}^{i} \geq \tilde{y}_{j}^{i}\right) \Longleftrightarrow\left(x^{i} \geq y^{i}\right),
$$

as desired, by applying first COLUMN PREFERENCES, then COORDINATE MONOTONICITY, and finally the definition of $\widetilde{\mathbf{X}}$ and $\widetilde{\mathbf{Y}}$.

Case 2. Now let $\mathcal{X}$ be any open convex set. Then the coordinate projection $\mathcal{X}_{j}$ is also open and convex, so the line segment $\mathcal{K}$ between $\mathbf{x}$ and $\mathbf{y}$ is in $\mathcal{X}_{j}$. For each $\mathbf{z} \in \mathcal{K}$, we can find an open box $\mathcal{B}_{\mathbf{z}} \subseteq \mathcal{X}_{j}$ that contains $\mathbf{z}$, and an open box $\widetilde{\mathcal{B}_{\mathbf{z}}} \subseteq \mathcal{X}$ that projects onto $\mathcal{B}_{\mathbf{z}}$. Apply the argument from Case 1 to $\widetilde{\mathcal{B}}_{\mathbf{z}}$ to show that $\succeq_{j}$ satisfies COLUMN MonOTONICITY when restricted to $\mathcal{B}_{\mathbf{z}}$. Since $\mathcal{K}$ is compact, it can be covered with a finite collection of boxes like $\mathcal{B}_{\mathbf{Z}}$, and $\succeq_{j}$ satisfies COLUMN Monotonicity on each one. An inductive argument leads one to conclude that $\mathbf{x} \succeq_{j} \mathbf{y}$ if and only if $x^{i} \geq y^{i}$.

The proof of Row Monotonicity is similar, only using Row PREFERENCES instead of COLUMN PREFERENCES.

Proof of Example (a) just above Proposition 1. Without loss of generality, we can take $\mathcal{X}=$ $(0,1)^{I \times J}$. Fix $\mathbf{X} \in \mathcal{X}$, letting $\mathcal{Y}:=\{\mathbf{Y} \in \mathcal{X} ; \mathbf{Y} \approx \mathbf{X}\}$. Given $\mathbf{Y}_{1}, \mathbf{Y}_{2} \in \mathcal{Y}$, we must find a path in $\mathcal{Y}$ connecting $\mathbf{Y}_{1}$ to $\mathbf{Y}_{2}$.

Define $\mathbf{1} \in \mathbb{R}^{I \times J}$ by setting $\mathbf{1}_{j}^{i}:=1$ for all $i \in I$ and $j \in J$. By Continuity and CoordiNATE Monotonicity, there exists $r_{\mathbf{1}} \in(0,1)$ such that $r_{\mathbf{1}} \mathbf{1} \in \mathcal{Y}$. Let $\mathcal{Z}_{1} \subset \mathcal{X}$ be the open line segment from $\mathbf{Y}_{1}$ to $\mathbf{1}$. For all $\mathbf{Z} \in \mathcal{Z}_{1}$, Coordinate Monotonicity implies that $\mathbf{Z} \succeq \mathbf{Y}_{1}$. Again by CONTINUity and CoORdinate Monotonicity, there exists $r_{\mathbf{Z}} \in(0,1]$ such that $r_{\mathbf{Z}} \mathbf{Z} \in \mathcal{Y}$. The set $\mathcal{K}_{1}:=\left\{r_{\mathbf{Z}} \mathbf{Z} ; \mathbf{Z} \in \mathcal{Z}_{1}\right\}$ is a continuous path in $\mathcal{Y}$ from $\mathbf{Y}_{1}$ to $r_{\mathbf{1}} \mathbf{1}$.

Likewise, a continuous path $\mathcal{K}_{2}$ can be found in $\mathcal{Y}$ from $\mathbf{Y}_{2}$ to $r_{\mathbf{1}} \mathbf{1}$. A path in $\mathcal{Y}$ from $\mathbf{Y}_{1}$ to $\mathbf{Y}_{2}$ results from joining it to $\mathcal{K}_{1}$. 
The proof of Proposition 1 is based on the Debreu-Gorman theory of additive representations for separable preference orders, which requires some background. Let $N$ be an indexing set (e.g., $N=I \times J)$, let $\mathcal{Y}$ be an open subset of $\mathbb{R}^{N}$, and for all $n \in N$, let $\mathcal{Y}_{n}$ be the projection of $\mathcal{Y}$ onto the $n$-th coordinate. A preference order $\succeq$ on $\mathcal{Y}$ has a fully additive representation if there exist functions $u_{n}: \mathcal{Y}_{n} \longrightarrow \mathbb{R}$, for all $n \in N$, such that if we define $U: \mathcal{Y} \longrightarrow \mathbb{R}$ by

$$
U(\mathbf{y}):=\sum_{n \in N} u_{n}\left(y_{n}\right)
$$

then $U$ represents $\succeq$.

For any $\mathbf{y} \in \mathcal{Y}$, we say that $\succeq$ admits a fully additive representation near $y$ if there is an open neighborhood $\mathcal{Y}^{\prime} \subseteq \mathcal{Y}$ around $\mathbf{y}$, such that $\succeq$ admits a fully additive representation when restricted to $\mathcal{Y}^{\prime}$. We will use the following result.

Lemma A1. Let $\mathcal{Y}$ be an open, connected, sectionally connected subset of $\mathbb{R}^{N}$, and let $\succeq$ be a continuous, indifference-connected preference order on $\mathcal{Y}$, which is strictly increasing in every coordinate. If $\succeq$ admits a fully additive representation near every $y \in \mathcal{Y}$, then $\succeq$ admits a fully additive representation on $\mathcal{Y}$. Furthermore, this global additive representation is unique up to a positive affine transformation.

Proof. See Theorem 2.2 of Chateauneuf and Wakker [9].

Let $J \subseteq N$, and let $K:=N \backslash J$. For any $\mathbf{y} \in \mathcal{Y}$, define $\mathbf{y}_{J}:=\left[y_{j}\right]_{j \in J}$ (an element of $\mathbb{R}^{J}$ ) and $\mathbf{y}_{K}:=\left[y_{k}\right]_{k \in K}$ (an element of $\mathbb{R}^{K}$ ). We say that $\succeq$ is $J$-separable (or that $J$ is a $\succeq$-separable subset of $N$ ) if the following holds. For all $\mathbf{x}, \mathbf{y}, \mathbf{x}^{\prime}, \mathbf{y}^{\prime} \in \mathcal{Y}$, if

$$
\begin{array}{lll}
\mathbf{x}_{K}=\mathbf{y}_{K}, & \mathbf{x}_{J}=\mathbf{x}_{J}^{\prime}, \\
\mathbf{x}_{K}^{\prime}=\mathbf{y}_{K}^{\prime}, & \text { and } & \mathbf{y}_{J}=\mathbf{y}_{J}^{\prime},
\end{array}
$$

then $(\mathbf{x} \succeq \mathbf{y}) \Longleftrightarrow\left(\mathbf{x}^{\prime} \succeq \mathbf{y}^{\prime}\right)$. We say that $\succeq$ is totally separable if every subset $J \subseteq N$ is $\succeq$-separable. A well-known result applies these concepts to the case where $\mathcal{Y}$ is an open box.

Lemma A2. If $\succeq$ is a continuous, totally separable preference order on an open box $\mathcal{B} \subseteq \mathbb{R}^{N}$, and $\succeq$ is increasing in every coordinate, then $\succeq$ has a fully additive utility representation.

Proof. See Theorem 3 in Debreu [10].

Let $J \subseteq N$ and $K:=N \backslash J$. We say that $J$ is strictly $\succeq$-essential if, for any $\mathbf{y} \in \mathcal{Y}$, there exist $\mathbf{x}, \mathbf{x}^{\prime} \in \mathcal{Y}$ such that $\mathbf{x}_{K}=\mathbf{x}_{K}^{\prime}=\mathbf{y}_{K}$, but $\mathbf{x} \succ \mathbf{x}^{\prime}$. (In words, it is possible to create a strict preference by only manipulating the $J$ coordinates, while keeping the $K$ coordinates fixed at any stipulated values.)

Lemma A3. Let $\succeq$ be a continuous preference order on an open box $\mathcal{B} \subseteq \mathbb{R}^{N}$. Let $J, K \subseteq N$ be two $\succeq$-separable subsets, such that $J \cap K \neq \emptyset$. Suppose that $J, K$, and $J \cap K$ are all strictly $\succeq$-essential. Then:

(a) $J \cup K$ is $\succeq$-separable.

(b) $J \cap K$ is $\succeq$-separable. 
Proof. See Theorem 1 by Gorman [17] for the original result, Theorem 4.7 of Blackorby et al. [4] for a restatement, and Theorem 11 and Proposition 16 of von Stengel [36] for the most general treatment.

Now, for any $i \in I$, define $J^{i}:=\{(i, j) ; j \in J\}$. We can write $\mathbb{R}^{I \times J}=\mathbb{R}^{J_{1}} \times \mathbb{R}^{J_{2}} \times \cdots \times \mathbb{R}^{J_{n}}$. For any $j \in J$, define $I_{j}:=\{(i, j) ; i \in I\}$. Similarly, we can write $\mathbb{R}^{I \times J}=\mathbb{R}^{I_{1}} \times \mathbb{R}^{I_{2}} \times \cdots \times \mathbb{R}^{I_{m}}$.

Lemma A4. As in Lemma A3, let $\succeq$ be a continuous preference order on an open box $\mathcal{B} \subseteq \mathbb{R}^{I \times J}$. For all $i \in I$ and $j \in J$, suppose the sets $I_{j}$ and $J^{i}$ are $\succeq$-separable, and the set $\{(i, j)\}$ is $\succeq$-strictly essential. Then $\succeq$ is totally separable.

Proof. Clearly, the union of two strictly $\succeq$-essential subsets of $I \times J$ is strictly essential. Since every singleton subset of $I \times J$ is strictly $\succeq$-essential, it follows that every subset of $I \times J$ is strictly $\succeq$-essential.

To show from the assumptions that $\succeq$ is totally separable, consider the cases of singleton and doubleton subsets of $I \times J$. Singletons $\{(i, j)\}$ are intersections of the $\succeq$-separable subsets $J^{i}$ and $I_{j}$, hence $\succeq$-separable by Lemma A3(b). A slightly more roundabout application of Lemma A3 shows that doubletons are $\succeq$-separable. Finally, prove that any subset $J \subseteq I \times J$ is $\succeq$-separable, by induction on $|J|$, doubleton separability, and Lemma A3(a). (See also Corollary to Theorem 3.7 in Keeney and Raiffa [24].)

Remark. To show that doubletons are separable in the proof of Lemma A4, we need $|I| \geq 2$ and $|J| \geq 2$. This is the key place in the proofs where this assumption is necessary.

Proof of Proposition 1(a). Given $\mathbf{X} \in \mathcal{X}$, there is an open box $\mathcal{B}$ of $\mathbb{R}^{I \times J}$ such that $\mathbf{X} \in \mathcal{B} \subseteq \mathcal{X}$. We first show that if $\succeq$ is restricted to $\mathcal{B}$, then it is $J^{i}$-separable for all $i \in I$. Let $\mathbf{Y}, \mathbf{Z}, \overline{\mathbf{Y}}, \overline{\mathbf{Z}} \in \mathcal{B}$, and suppose that (a) $\bar{y}^{h}=\mathbf{z}^{h}$ for all $h \in I \backslash\{i\}$, (b) $\mathbf{y}^{i}=\overline{\mathbf{y}}^{i}$, (c) $\overline{\mathbf{y}}^{h}=\overline{\mathbf{z}}^{h}$ for all $h \in I \backslash\{i\}$, and (d) $\mathbf{z}^{i}=\overline{\mathbf{z}}^{i}$. Then

$$
(\mathbf{Y} \succeq \mathbf{Z}) \quad \Longleftrightarrow \quad\left(\mathbf{y}^{i} \succeq^{i} \mathbf{z}^{i}\right) \quad \Longleftrightarrow \quad\left(\overline{\mathbf{y}}^{i} \succeq^{i} \overline{\mathbf{z}}^{i}\right) \quad \Longleftrightarrow \quad(\overline{\mathbf{Y}} \succeq \overline{\mathbf{Z}}),
$$

showing that $\succeq$ is $J^{i}$-separable. (The first equivalence is by (a) and Row PREFERENCES, the second by (b) and (d), and the last one by (c) and Row PREFERENCES.)

By a similar argument based on COLUMn PREFEREnCES, if $\succeq$ is restricted to $\mathcal{B}$, then it is $I_{j}$-separable for all $j \in J$.

It remains to show that $\succeq$ has a fully additive representation on $\mathcal{B}$. By CONTINUITY, $\succeq$ is continuous on $\mathcal{B}$. COORDINATE MONOTONICITY implies that every coordinate is strictly essential. We have just shown that $J_{i}$ and $I_{j}$ are separable for all $i$ and $j$; thus Lemma A4 implies that $\succeq$ is totally separable on $\mathcal{B}$. Finally, Lemma A2 and COORDINATE MONOTONICITY yield an additive representation of $\succeq$ on $\mathcal{B}$. This proves part (a) with $\mathcal{Y}=\mathcal{B}$.

Proof of (b). This follows from part (a), along with CoORdinATE MONOTONICITY, ContinuITY and Lemma A1. ${ }^{25}$ (Alternatively, we could have directly proved (b) by applying Theorem 1 of Segal [32].)

$\overline{25}$ This is the one place in the proof that makes use of sectional connectedness and indifference connectedness. 
Proof of (d). Fix $\mathbf{X} \in \mathcal{X}$, and consider the section of $\mathcal{X}$ in the $j$ th dimension through $\mathbf{X}$, as defined by:

$$
\mathcal{S}_{j}(\mathbf{X}):=\left\{\mathbf{Y} \in \mathcal{X} ; \mathbf{y}_{k}=\mathbf{x}_{k}, \text { for all } k \in J \backslash\{j\}\right\} .
$$

Let $\mathcal{X}_{j}(\mathbf{X}):=\left\{\mathbf{y}_{j} ; \mathbf{Y} \in \mathcal{S}_{j}(\mathbf{X})\right\} \subseteq \mathcal{X}_{j}$. Column Preferences implies that $\succeq$, when restricted to $\mathcal{S}_{j}(\mathbf{X})$, is equivalent to $\succeq_{j}$ on $\mathcal{X}_{j}(\mathbf{X})$. Thus, part (b) implies that the order $\succeq_{j}$ on $\mathcal{X}_{j}(\mathbf{X})$ is represented by the function $U_{j}^{\mathbf{X}}$ defined by

$$
U_{j}^{\mathbf{X}}(\mathbf{y}):=\overbrace{\sum_{k \in J \backslash\{j\}} \sum_{i \in I} u_{k}^{i}\left(x_{k}^{i}\right)}^{\mathrm{a} \text { constant }}+\sum_{i \in I} u_{j}^{i}\left(y^{i}\right),
$$

for all $\mathbf{y} \in \mathcal{X}_{j}(\mathbf{X})$. Thus, the function $U_{j}:=\sum_{i \in I} u_{j}^{i}\left(y^{i}\right)$ also represents $\succeq_{j}$ on $\mathcal{X}_{j}(\mathbf{X})$. This holds for all $\mathbf{X} \in \mathcal{X}$; thus $U_{j}$ represents $\succeq_{j}$ on $\mathcal{X}_{j}=\bigcup_{\mathbf{X} \in \mathcal{X}} \mathcal{X}_{j}(\mathbf{X})$.

Proof of (c). Similar to the proof of (d), only using Row PREFERENCES instead of COLUMN PREFERENCES.

To prove Theorem 1, we must solve a Pexider functional equation on a general domain. The solution is provided by the following result.

Lemma A5. Let $N \geq 1$ be an integer, and let $\mathcal{Y} \subseteq \mathbb{R}^{N}$ be an open, connected set. For all $n \in$ $[1 \ldots N]$, let $\mathcal{Y}_{n}$ be the projection of $\mathcal{Y}$ onto the nth coordinate, and let $\mathcal{Y}_{0}:=\left\{\sum_{n=1}^{N} y_{n} ; \mathbf{y} \in \mathcal{Y}\right\} .{ }^{26}$ For all $n \in[0 \ldots N]$, let $f_{n}: \mathcal{Y}_{n} \longrightarrow \mathbb{R}$ be functions, at least one of which is continuous, and suppose they satisfy the Pexider equation:

$$
f_{0}\left(\sum_{n=1}^{N} y_{n}\right)=\sum_{n=1}^{n} f_{n}\left(y_{n}\right), \quad \text { for all } \mathbf{y} \in \mathcal{Y} \text {. }
$$

Then there exist (unique) constants $a, b_{0}, b_{1}, b_{2}, \ldots, b_{N} \in \mathbb{R}$ such that $b_{0}=\sum_{n=1}^{N} b_{n}$, and such that, for all $n \in[0 \ldots N], f_{n}(y)=a y+b_{n}$ for all $y \in \mathcal{Y}_{n}$.

Proof. See Theorem 1 and Corollary 2 in Radó and Baker [30].

Proof of Theorem 1. The "if" direction is obvious. We prove the "only if" direction.

Proof of (a). This follows from adapting the representations in Proposition 1(d) to the fact that $\mathcal{X}$ now satisfies Identical Column Spaces and $\succeq$ now satisfies InVARIANT COLUMN PREFERENCES. (Specifically, fix some $j_{0} \in J$, and for all $i \in I$, and all $\mathbf{x} \in \mathcal{X}_{J}$, define $u^{i}\left(x^{i}\right):=u_{j_{0}}^{i}\left(x^{i}\right)$, and put $W_{J}=U_{j_{0}}$, where $U_{j_{0}}$ is defined by setting $j=j_{0}$ in Proposition $1(\mathrm{~d})$.)

To prove parts (b)-(d), fix some $j_{0} \in J$. For all $i \in I$, let $u^{i}:=u_{j_{0}}^{i}$.

Claim 1. For any $j \in J$, there exist constants $a_{j}>0$ and $b_{j}^{i} \in \mathbb{R}$ such that $u_{j}^{i}\left(x^{i}\right)=a_{j} u^{i}\left(x^{i}\right)+b_{j}^{i}$ for all $\mathbf{x} \in \mathcal{X}_{J}$ and $i \in I$.

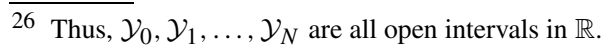


Proof. By Identical Column Spaces, $\mathcal{X}_{J}$ is the same as $\mathcal{X}_{j}$ for any $j \in J$, so it is an open and connected set of $\mathbb{R}^{I}$ by the usual properties of the projection map. Let $j \in J$, and let $U_{j_{0}}$ and $U_{j}$ be as in Proposition 1(d). By InVARIAnt Column PREFERENCES, both $U_{j_{0}}$ and $U_{j}$, represent $\succeq_{J}$ on $\mathcal{X}_{J}$. Thus, there are continuous, increasing transformations $g_{j}: \mathbb{R} \longrightarrow \mathbb{R}$ such that $U_{j}=$ $g_{j} \circ U_{j 0}$, or

$$
\sum_{i \in I} u_{j}^{i}\left(x^{i}\right)=g_{j}\left(\sum_{i \in I} u_{j_{0}}^{i}\left(x^{i}\right)\right), \quad \text { for all } \mathbf{x} \in \mathcal{X}_{J} .
$$

For simplicity, suppose $I=\{1, \ldots, n\}$, so that $\mathcal{X}_{J} \subseteq \mathbb{R}^{n}$. The image set $\mathcal{Z}:=\left\{\left(u_{1}^{1}\left(x^{1}\right), \ldots\right.\right.$, $\left.\left.u_{1}^{n}\left(x^{n}\right)\right) ; \mathbf{x} \in \mathcal{X}_{J}\right\}$ is also open and connected in $\mathbb{R}^{n}$, because the $u_{j_{0}}^{i}$ are continuous and increasing, hence open. ${ }^{27}$ If we make the change of variables $z^{i}:=u_{j_{0}}^{i}\left(x^{i}\right)$ for all $i \in I$, then (A1) becomes the Pexider equation:

$$
\sum_{i \in I} u_{j}^{i} \circ\left(u_{j_{0}}^{i}\right)^{-1}\left(z^{i}\right)=g_{j}\left(\sum_{i \in I} z^{i}\right), \quad \text { for all } \mathbf{z} \in \mathcal{Z} .
$$

Lemma A5 applied to $\mathcal{Z}$ yields constants $a_{j}$ and $b_{j}^{1}, \ldots, b_{j}^{n} \in \mathbb{R}$ such that $u_{j}^{i} \circ\left(u_{1}^{i}\right)^{-1}\left(z^{i}\right)=$ $a_{j} z^{i}+b_{j}^{i}$ for all $\mathbf{z} \in \mathcal{Z}$ and all $i \in I$, hence such that $u_{j}^{i}\left(x^{i}\right)=a_{j} u_{j_{0}}^{i}\left(x^{i}\right)+b_{j}^{i}$ for any $\mathbf{x} \in \mathcal{X}_{J}$. Finally, $a_{j}>0$ because $u_{j}^{i}$ and $u_{j_{0}}^{i}$ are both increasing. $\square$ Claim 1

Proof of (c). Let $A:=\sum_{j \in J} a_{j}$ and $p_{j}:=a_{j} / A$ for all $j \in J$, so that $\mathbf{p}=\left(p_{j}\right)_{j \in J}$ is a strictly positive weight vector on $J$. Claim 1 implies that, for all $i \in I$ and $j \in J$, and all $\mathbf{X} \in \mathcal{X}$,

$$
u_{j}^{i}\left(x_{j}^{i}\right)=A p_{j} u^{i}\left(x_{j}^{i}\right)+b_{j}^{i} .
$$

If we let $U: \mathcal{X} \longrightarrow \mathbb{R}$ be as in Proposition $1(\mathrm{a}, \mathrm{b})$, and define $B:=\sum_{i \in I} \sum_{j \in J} b_{j}^{i}$, then for all $\mathbf{X} \in \mathcal{X}$

$$
\begin{aligned}
U(\mathbf{X}) & =\sum_{i \in I} \sum_{j \in J} u_{j}^{i}\left(x_{j}^{i}\right)=A \cdot \sum_{i \in I} \sum_{j \in J} p_{j} u^{i}\left(x_{j}^{i}\right)+\sum_{i \in I} \sum_{j \in J} b_{j}^{i} \\
& =A \cdot \sum_{j \in J} p_{j}\left(\sum_{i \in I} u^{i}\left(x_{j}^{i}\right)\right)+B=A \cdot W(\mathbf{X})+B,
\end{aligned}
$$

where $W$ is defined as in Eq. (3). Thus, $W$ is an increasing transform of $U$, so it represents $\succeq$ on $\mathcal{X}$.

Proof of (b). Let $U^{i}$ be as in Proposition 1(c). Then for all $\mathbf{x} \in \mathcal{X}^{i}$,

$$
U^{i}(\mathbf{x})=\sum_{j \in J} u_{j}^{i}\left(x_{j}\right)=A \sum_{j \in J} p_{j} u^{i}\left(x_{j}\right)+\sum_{j \in J} b_{j}^{i}=A U_{\mathbf{p}}^{i}(\mathbf{x})+[\text { a constant }],
$$

where the second equality is by (A2). Thus, $U_{\mathbf{p}}^{i}$ represents $\succeq^{i}$.

\footnotetext{
27 Any function $\phi$ from an open subset of $\mathbb{R}$ to $\mathbb{R}$ that is continuous and increasing is also open. We will make repeated use of this property.
} 
Proof of (d). For all $i \in I$, let $\widetilde{u}^{i}: \mathbb{R} \longrightarrow \mathbb{R}$ be a continuous and increasing function, and let $\widetilde{\mathbf{p}} \in \Delta_{J}$ be a strictly positive weight vector. Suppose that $\succeq_{J}$ is represented by the function $\widetilde{W}_{J}: \mathcal{X}_{J} \longrightarrow \mathbb{R}$ defined by

$$
\widetilde{W}_{J}(\mathbf{x}):=\sum_{i \in I} \widetilde{u}^{i}\left(x^{i}\right), \quad \text { for all } \mathbf{x} \in \mathcal{X}_{J},
$$

and that $\succeq$ is also represented by the function $\widetilde{W}: \mathcal{X} \longrightarrow \mathbb{R}$ defined by

$$
\widetilde{W}(\mathbf{X}):=\sum_{j \in J} \sum_{i \in I} \tilde{p}_{j} \tilde{u}^{i}\left(x_{j}^{i}\right), \quad \text { for all } \mathbf{X} \in \mathcal{X} .
$$

Now, $\sum_{i \in I} \widetilde{u}^{i}\left(x^{i}\right)=g\left(\sum_{i \in I} u^{i}\left(x^{i}\right)\right)$ for some increasing and continuous transformation $g .{ }^{28}$ Thus carrying the same functional equation argument as for Claim 1, we conclude that there are constants $a>0$ and $b^{i} \in \mathbb{R}$ (for all $i \in I$ ) such that

$$
\tilde{u}^{i}\left(x^{i}\right)=a u^{i}\left(x^{i}\right)+b^{i},
$$

for all $i \in I$ and $\mathbf{x} \in \mathcal{X}_{J}$. Thus, the functions $\left\{u^{i}\right\}_{i \in I}$ are unique up to a common affine transformation, as was to be proved.

Meanwhile, the uniqueness part of Proposition 1(a) yields constants $A>0$ and $b_{j}^{i} \in \mathbb{R}$, for all $i \in I$ and $j \in J$, such that

$$
\widetilde{p}_{j} \tilde{u}^{i}\left(x_{j}^{i}\right)=A p_{j} u^{i}\left(x_{j}^{i}\right)+b_{j}^{i},
$$

for all $\mathbf{X} \in \mathcal{X}, i \in I$ and $j \in J$. Fix some $i_{0} \in I$ Let $\mathbf{x} \in \mathcal{X}^{i_{0}}$. The set $\mathcal{X}^{i_{0}}$ is open, and $u^{i_{0}}$ is continuous and increasing; thus, there exist some $\varepsilon>0$ and some $\mathbf{y} \in \mathcal{X}^{i_{0}}$ such that $u^{i_{0}}\left(x_{j}\right)-$ $u^{i_{0}}\left(y_{j}\right)=\varepsilon$ for all $j \in J$. But then, for all $j \in J$,

$$
\begin{aligned}
a \varepsilon \tilde{p}_{j} & =a \tilde{p}_{j} u^{i_{0}}\left(x_{j}\right)-a \tilde{p}_{j} u^{i_{0}}\left(y_{j}\right)=\widetilde{p}_{j} \tilde{u}^{i_{0}}\left(x_{j}\right)-\tilde{p}_{j} \tilde{u}^{i_{0}}\left(y_{j}\right) \quad \text { (by Eq. (A3)) } \\
& =A p_{j} u^{i_{0}}\left(x_{j}\right)-A p_{j} u^{i_{0}}\left(y_{j}\right)=A \varepsilon p_{j}, \quad \text { (by Eq. (A4)). }
\end{aligned}
$$

It follows that $a \varepsilon \widetilde{\mathbf{p}}=A \varepsilon \mathbf{p}$, and thus $A=a$, since $\mathbf{p}$ and $\widetilde{\mathbf{p}}$ are weight vectors. Thus, $\mathbf{p}=\widetilde{\mathbf{p}}$, which completes the proof of $(\mathrm{d})$.

Proof of Proposition 2. (a) Let $W: \mathcal{X} \longrightarrow \mathbb{R}$ be the additive representation from Theorem 1(c). By hypothesis, for all $j \in\{1, \ldots, t-1\}$, there is some $\mathbf{X} \in \mathcal{X}$ such that $\mathbf{x}_{j} \succ_{J} \mathbf{x}_{j+1}$ and $\mathbf{X} \succ \sigma_{j}(\mathbf{X})$, so that $W(\mathbf{X})>W\left[\sigma_{j}(\mathbf{X})\right]$. Canceling the common summands from both of this inequality yields

$$
p_{j} W_{J}\left(\mathbf{x}_{j}\right)+p_{j+1} W_{J}\left(\mathbf{x}_{j+1}\right)>p_{j} W_{J}\left(\mathbf{x}_{j+1}\right)+p_{j+1} W_{J}\left(\mathbf{x}_{j}\right) .
$$

Rearranging this inequality, we obtain

$$
\left(p_{j}-p_{j+1}\right) \cdot\left[W_{J}\left(\mathbf{x}_{j}\right)-W_{J}\left(\mathbf{x}_{j+1}\right)\right]>0 .
$$

But $\mathbf{x}_{j} \succ_{J} \mathbf{x}_{j+1}$, so $W_{J}\left(\mathbf{x}_{j}\right)>W_{J}\left(\mathbf{x}_{j+1}\right)$. Thus, we must have $p_{j}>p_{j+1}$, as desired.

\footnotetext{
28 If $f$ and $h$ are continuous real-valued functions on some connected subset $B \subseteq \mathbb{R}$, and $g$ is an increasing real-valued function such that $h=g \circ f$, then $g$ is continuous on $f(B)$. We will make repeated use of this fact.
} 
(b) Theorem 1 implies that $\succeq_{L}$ on $\mathcal{X}_{L}$ and $\succeq_{R}$ on $\mathcal{X}_{R}$ have the additive representations:

$$
\begin{aligned}
& W_{L}(\mathbf{X})=\sum_{j=1}^{t-1} \sum_{i \in I} p_{j} u^{i}\left(x_{j}^{i}\right) \quad \text { and } \\
& W_{R}(\mathbf{X})=\sum_{j=2}^{t} \sum_{i \in I} p_{j} u^{i}\left(x_{j}^{i}\right) .
\end{aligned}
$$

On $\mathcal{X}_{L} \cap \mathcal{X}_{R} \neq \emptyset, W_{L}$ and $W_{R}$ represent the same order (by STATIONARITY). This intersection is open in $\mathbb{R}^{|I| \times(t-1)}$ (as $\mathcal{X}_{L}$ and $\mathcal{X}_{R}$ are open), and a standard uniqueness theorem for additive representations entails that $W_{L}$ and $W_{R}$ are identical up to a positive affine transformation. Thus, there is $\delta>0$ such that $\left(p_{2}, \ldots, p_{t}\right)=\delta \cdot\left(p_{1}, \ldots, p_{t-1}\right)$. Now, repeating the argument of part (a) for the given $j$, we have that $p_{j}>p_{j+1}$, hence $\delta<1$.

Proof of Corollary 1. Again, we prove the "only if" direction. Theorem 1(c) says that $\succeq$ is represented by the function $W: \mathcal{X} \longrightarrow \mathbb{R}$ defined by Eq. (3). Now, by the variant of this theorem using InVARIANT Row PREFERENCES and Identical Row Spaces, there is a weight vector $\mathbf{q}=$ $\left(q^{i}\right)_{i \in I} \in \Delta_{I}$, and, for all $j \in J$, there is an increasing, continuous function $v_{j}: \mathcal{X}_{j}^{*} \longrightarrow \mathbb{R}$, such that $\succeq^{I}$ is represented by the function $W^{I}: \mathcal{X} \longrightarrow \mathbb{R}$ defined by

$$
W^{I}(\mathbf{x}):=\sum_{j \in J} v_{j}\left(x_{j}\right), \quad \text { for all } \mathbf{x} \in \mathcal{X}^{I},
$$

while $\succeq$ is represented by the function $\widetilde{W}: \mathcal{X} \longrightarrow \mathbb{R}$ defined by

$$
\widetilde{W}(\mathbf{X}):=\sum_{j \in J} \sum_{i \in I} q^{i} v_{j}\left(x_{j}^{i}\right), \quad \text { for all } \mathbf{X} \in \mathcal{X} .
$$

Now fix $x_{0} \in \mathcal{X}_{*}^{*}$. By Theorem 1(d) and its variant, we can subtract relevant constants from the functions $\left\{v_{j}\right\}_{j \in J}$ and $\left\{u^{i}\right\}_{i \in I}$, to ensure that

$$
v_{j}\left(x_{0}\right)=0 \quad \text { for all } j \in J, \quad \text { and } \quad u^{i}\left(x_{0}\right)=0 \quad \text { for all } i \in I .
$$

Since $\succeq$ is represented by both $W$ and $\widetilde{W}$, there is some continuous, increasing function $f: \mathbb{R} \longrightarrow \mathbb{R}$ such that:

$$
f\left(\sum_{j \in J} \sum_{i \in I} p_{j} u^{i}\left(x_{j}^{i}\right)\right)=\sum_{j \in J} \sum_{i \in I} q^{i} v_{j}\left(x_{j}^{i}\right), \quad \text { for all } \mathbf{X} \in \mathcal{X} .
$$

For all $i \in I$ and $j \in J$, define $g_{j}^{i}(\zeta):=q^{i} v_{j} \circ\left(u^{i}\right)^{-1}\left(\zeta / p_{j}\right)$ for all $\zeta \in \mathbb{R}$ where this definition makes sense. Define $\Xi:=\left\{\left[p_{j} u^{i}\left(x_{j}^{i}\right)\right]_{j \in J}^{i \in I} ; \mathbf{X} \in \mathcal{X}\right\}$, an open, connected subset of $\mathbb{R}^{I \times J}$. Then substituting $\xi_{j}^{i}:=p_{j} u^{i}\left(x_{j}^{i}\right)$ into both sides of Eq. (A8) yields

$$
f\left(\sum_{j \in J} \sum_{i \in I} \xi_{j}^{i}\right)=\sum_{j \in J} \sum_{i \in I} g_{j}^{i}\left(\xi_{j}^{i}\right), \quad \text { for all } \xi \in \Xi .
$$

Now Lemma A5 implies that there exists a constant $a>0$ such that $f(\zeta)=a \zeta=g_{j}^{i}(\zeta)$ for all $i \in I$ and $j \in J$. (Eq. (A7) implies that the added constants of Lemma A5 are all 0.) By rescaling $\left\{v_{j}\right\}_{j \in J}$ if necessary, we can assume that $a=1$; hence $g_{j}^{i}(\zeta)=\zeta$. But $g_{j}^{i}(\zeta)=q^{i} v_{j}$ 。 
$\left(u^{i}\right)^{-1}\left(\zeta / p_{j}\right)$, so this implies that $p_{j} u^{i}=q^{i} v_{j}$, for all $(i, j) \in I \times J$. Dividing these equations by $q^{i} p_{j}$ (which are nonzero), we obtain

$$
u^{i} / q^{i}=v_{j} / p^{j}, \quad \text { for all }(i, j) \in I \times J .
$$

It follows that there is a single increasing continuous function $u: \mathcal{X}_{*}^{*} \longrightarrow \mathbb{R}$ such that

$$
\text { (a) } u^{i} / q^{i}=u \quad \text { for all } i \in I \text { and } \quad \text { (b) } v_{j} / p_{j}=u \text { for all } j \in J \text {. }
$$

Substituting Eq. (A9)(a) into Eq. (1) yields part (a) of the result. Substituting (A9)(b) into (A5) yields part (b), while substituting (A9)(b) into (A6) yields part (c). Part (d) is straightforward.

\section{References}

[1] F.J. Anscombe, R.J. Aumann, A definition of subjective probability, Ann. Math. Stat. 34 (1963) 199-205.

[2] C. Blackorby, D. Donaldson, P. Mongin, Social aggregation without the expected utility hypothesis, Discussion paper, Cahier du Laboratoire d'économétrie, Ecole Polytechnique, 2004.

[3] C. Blackorby, D. Donaldson, J.A. Weymark, Harsanyi's social aggregation theorem for state-contingent alternatives, J. Math. Econ. 32 (1999) 365-387.

[4] C. Blackorby, D. Primont, R. Russell, Duality, Separability, and Functional Structure: Theory and Economic Applications, North-Holland, New York, 1978.

[5] H. Bleichrodt, K. Rohde, P. Wakker, Koopmans' constant discounting for intertemporal choice: a simplification and a generalization, J. Math. Psychol. 52 (2008) 341-347.

[6] J. Broome, Weighing Goods, Basic Blackwell, Oxford, UK, 1991.

[7] M. Browning, A simple nonadditive preference structure for models of household behavior over time, J. Polit. Economy 99 (1991) 607-637.

[8] C.P. Chambers, T. Hayashi, Preference aggregation under uncertainty: Savage vs Pareto, Games Econ. Behav. 54 (2006) 430-440.

[9] A. Chateauneuf, P. Wakker, From local to global additive representation, J. Math. Econ. 22 (1993) 523-545.

[10] G. Debreu, Topological methods in cardinal utility theory, in: Mathematical Methods in the Social Sciences 1959 , Stanford Univ. Press, Stanford, Calif., 1960, pp. 16-26.

[11] P.C. Fishburn, Utility Theory for Decision Making, Publ. Oper. Res. Ser., vol. 18, John Wiley \& Sons Inc., New York, 1970.

[12] M. Fleurbaey, Two variants of Harsanyi's aggregation theorem, Econ. Letters 105 (2009) 300-302.

[13] T. Gajdos, J.-M. Tallon, J.-C. Vergnaud, Representation and aggregation of preferences under uncertainty, J. Econ. Theory 141 (2008) 68-99.

[14] I. Gilboa, D. Samet, D. Schmeidler, Utilitarian aggregation of beliefs and tastes, J. Polit. Economy 112 (2004) 932-938.

[15] M. Gold, J. Siegel, L. Russell, M. Weinstein, Cost-Effectiveness in Health and Medicine, Oxford University Press, New York, 1996.

[16] W. Gorman, Conditions for additive separability, Econometrica 36 (1968) 605-609.

[17] W. Gorman, The structure of utility functions, Rev. Econ. Stud. 35 (1968) 367-390.

[18] H. Green, Aggregation in Economic Analysis, Princeton University Press, Princeton, 1964.

[19] P.J. Hammond, Ex ante and ex post welfare optimality under uncertainty, Economica 48 (1981) 235-250.

[20] P.J. Hammond, Utilitarianism, uncertainty and information, in: A. Sen, B. Williams (Eds.), Utilitarianism and Beyond, Cambridge University Press, Cambridge, 1983, pp. 85-102.

[21] J.C. Harsanyi, Cardinal welfare, individualistic ethics, and interpersonal comparisons of utility, J. Polit. Economy 63 (1955) 309-321.

[22] R.L. Keeney, Analysis of preference dependencies among objectives, Oper. Res. 29 (1981) 1105-1120.

[23] R.L. Keeney, R. Nau, A theorem for Bayesian group decisions, J. Risk Uncertainty 43 (2011) 1-17.

[24] R.L. Keeney, H. Raiffa, Decisions with Multiple Objectives: Preferences and Value Tradeoffs, John Wiley \& Sons, New York, 1976, republished by Cambridge University Press, 1993.

[25] T. Koopmans, Stationary ordinal utility and impatience, Econometrica 28 (1960) 287-309.

[26] P. Mongin, Consistent Bayesian aggregation, J. Econ. Theory 66 (1995) 313-351.

[27] P. Mongin, The paradox of the Bayesian experts and state-dependent utility theory, J. Math. Econ. 29 (1998) $331-361$. 
[28] P. Mongin, C. d'Aspremont, Utility theory and ethics, in: S. Barberà, P. Hammond, C. Seidl (Eds.), Handbook of Utility Theory, vol. 1, Kluwer, 1998, pp. 371-481, Chap. 10.

[29] A. Nataf, Sur la possibilité de construction de certains macromodèles, Econometrica 16 (1948) $232-244$.

[30] F. Radó, J.A. Baker, Pexider's equation and aggregation of allocations, Aequ. Math. 32 (1987) 227-239.

[31] L.J. Savage, The Foundations of Statistics, Dover Publications Inc., New York, 1972.

[32] U. Segal, Additively separable representations on nonconvex sets, J. Econ. Theory 56 (1992) 89-99.

[33] R. Starr, Optimal production and allocation under uncertainty, Quart. J. Econ. 87 (1973) 81-95.

[34] J. van Daal, A. Merkies, Aggregation in Economic Research: From Individual to Macro Relations, Reidel, Dordrecht, 1984.

[35] J. van Daal, A. Merkies, The problem of aggregation of individual economic relations. Consistency and representativity in historical perspective, in: W. Eichhorn (Ed.), Measurement in Economics, Physica-Verlag, Heidelberg, 1988, pp. 607-637.

[36] B. von Stengel, Closure properties of independence concepts for continuous utilities, Math. Oper. Res. 18 (1993) 346-389.

[37] P. Wakker, Additive representations on rank-ordered sets. II. The topological approach, J. Math. Econ. 22 (1993) $1-26$.

[38] P.P. Wakker, Additive Representations of Preferences, Kluwer, Dordrecht, 1989.

[39] J. Williamson, In Defence of Objective Bayesianism, Oxford University Press, Oxford, 2010. 